\title{
LA-UR-12-26876
}

Approved for public release; distribution is unlimited.

Title:

Author(s):

Intended for:

\section{Multiscale Image Decomposition and Analysis of Shapes}

\author{
Prasad, Lakshman
}

Invited Talk at the Woods Hole Oceanographic Institution

\section{- LOSAlamos

\author{
EST. 1943
}

Disclaimer:

Los Alamos National Laboratory, an affirmative action/equal opportunity employer,is operated by the Los Alamos National

Security, LLC for the National NuclearSecurity Administration of the U.S. Department of Energy under contract DE-AC52-06NA25396.

By approving this article, the publisher recognizes that the U.S. Government retains nonexclusive, royalty-free license to

publish or reproduce the published form of this contribution, or to allow others to do so, for U.S. Government purposes.

Los Alamos National Laboratory requests that the publisher identify this article as work performed under the auspices of the

U.S. Departmentof Energy. Los Alamos National Laboratory strongly supports academic freedom and a researcher's right to publish; as an institution, however, the Laboratory does not endorse the viewpoint of a publication or guarantee its technical correctness. 
Woods Hole Oceanographic Institution AOPE Seminar 12/19/12

Multiscale Image Decomposition and.

Analysis of Shapes

Lakshman Prasad prasad@lani.gov s:

Space Data Systems Group Intelligence and Space Research Division

4. Los Alamos National Laboratory, Los Alamos, NM 


\section{Image Understanding: Two key tasks}

Image Segmentation:

pixels $\Rightarrow \ldots \ldots \ldots \Rightarrow$ objects

Object Recognition:

objects $\Rightarrow$........ $\Rightarrow$ Jecognition 


\section{In search of a framework}

\section{A successful visual processing system}

- Must support abstraction

capable of going from a low-level to high-level of representation

- Must be open-ended

support multiple visual routines

- Must be able to handle complexity

should deal with real world imagery

Shimon Ullman

It must also be:

Efficient, Economical, Perceptually relevant

The human visual system is all this

How can we mimic it algorithmically? 


\section{What is really important in an image?}

Image discontinuities (edges)

- are more informative than regularities (perceptual)

- are a much smaller subset (economical)

\section{How can we synthesize edge information into meaningful objects?}

Elder and Goldberg studied the statistics of contour grouping cues used by humans in natural images and found that the law of proximity is by far the single most powerful cue for contour grouping.

J. H. Elder and R. M. Goldberg, "Ecological statistics of gestalt laws for the perceptual organization of contours," Journal of Vision, vol. 2, no. 4, pp. 324-353, 52002. 
ENTER: Delaunay triangulation; Mother of all proximity graphs

Defn: A triangulation of a point set where the interior of the circumcircle of each triangle is empty

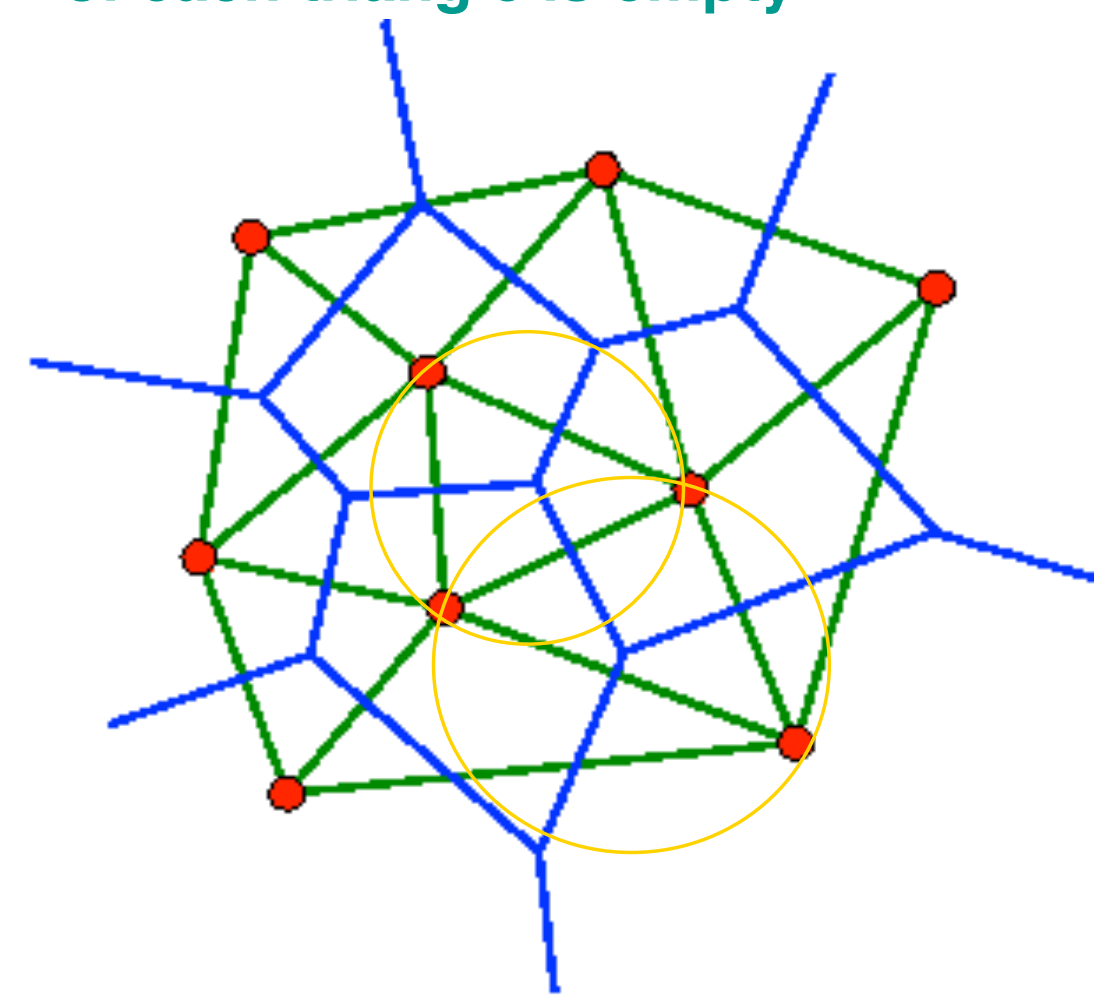

Other properties:

-Maximizes minimum angle over all triangles

- Sum of angles across any edge $\leq 180^{\circ}$

- It is the geometric dual of the Voronoi diagram of the point set

$\mathrm{NNG} \subseteq \mathrm{MST} \subseteq \mathrm{RNG} \subseteq \mathrm{G}_{\beta} \subseteq \mathrm{GG} \subseteq \mathrm{DT}$

The Delaunay Trlangulation (green) of a polnt set (red) and Its Voronol Dlagram (blue) 


\section{Can Delaunay triangulation be used to complete contours?}

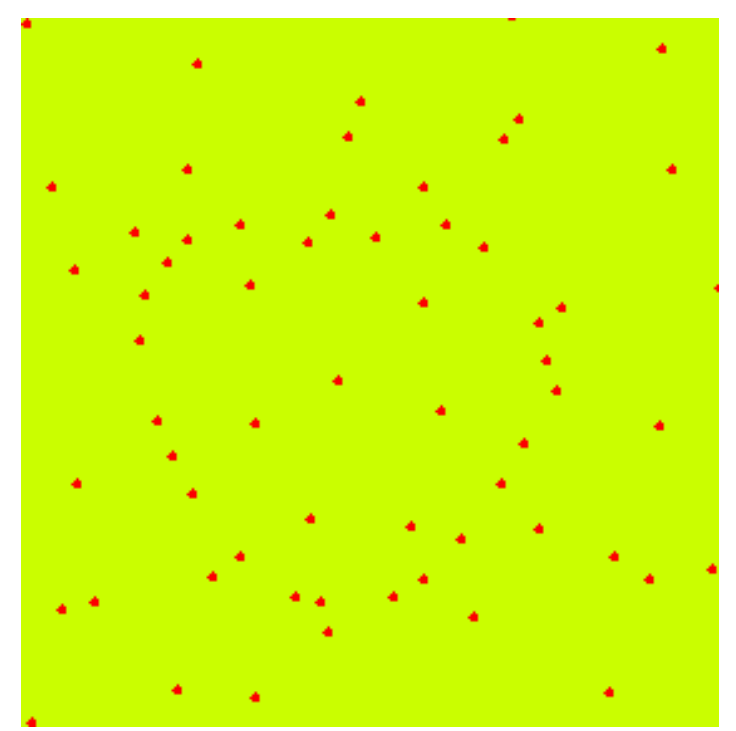

1a) Apple in noise

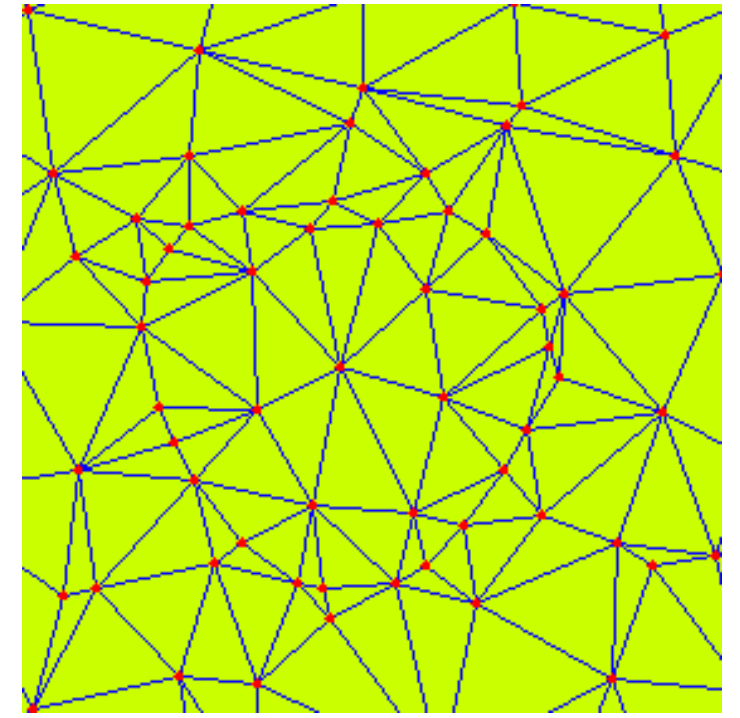

1b) Delaunay Triangulation

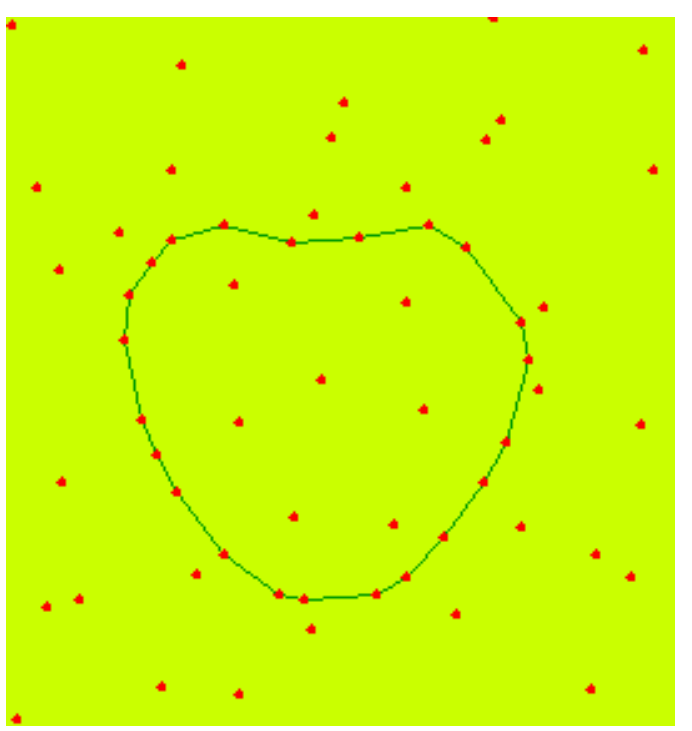

1c) Apple contour 


\section{Can Delaunay triangulation reconstruct images from edges?}

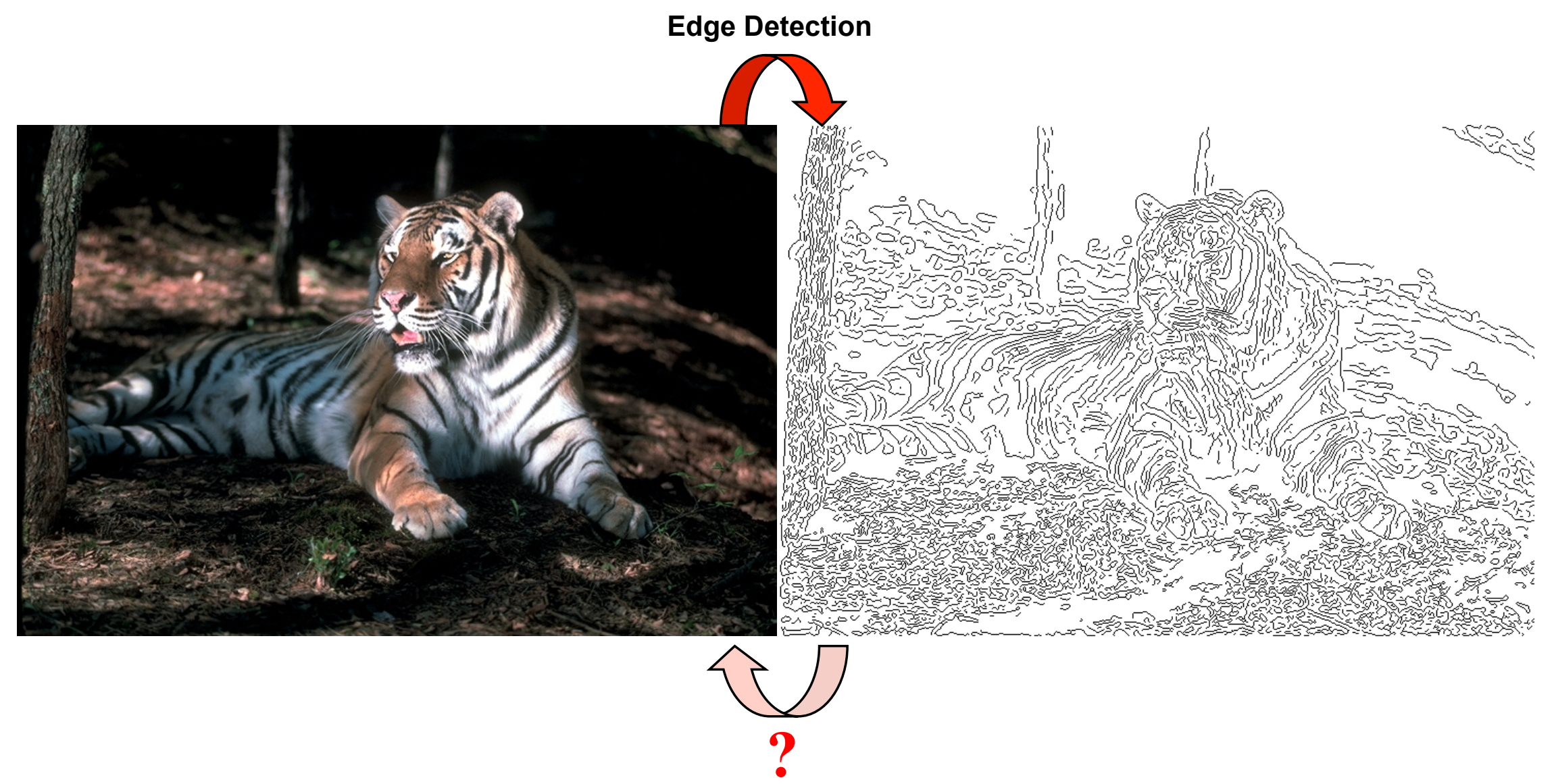




\section{Pixels to Trixels:}

\section{towards structural image segmentation}

The Delaunay triangulation of an image' s edge pixels is a featureadaptive proximity grid providing regional bindings between contour elements.

The Delaunay grid is a fertile field for perceptually desirable contour completions

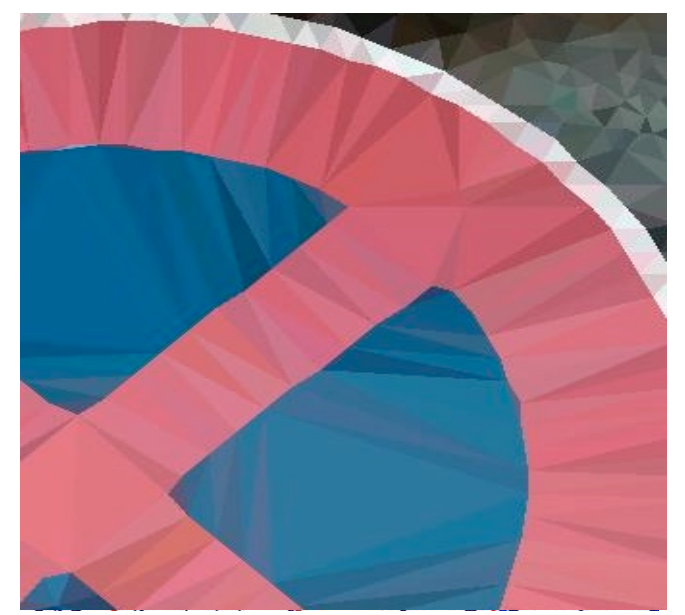

To each triangle we attribute the average color of the pixels in its interior, and call it a triangular excision element or trixel. 


\section{Modeling Perceptual Organization}

An edge gets a value of 1 if it satisfies an edge continuation perceptual criterion such as collinearity, transversality, or closure.

An edge gets a value of 0 if it satisfies a region continuation perceptual criterion such as common color, common boundary, parallelism, or symmetry.

Finally, an edge is retained (closed) or deleted (open) based on whether it satisfies a composite perceptual organization criterion that is a Boolean expression in some elementary perceptual criteria:

$$
\mathrm{C}=\left(p_{1} \&\left(p_{2}\left|\left(p_{4} \&\left(\sim p_{5} \mid p_{6}\right)\right)\right| \ldots \mid\left(p_{n-1} \& \sim p_{n}\right)\right)\right.
$$

A trixel edge $e$ is a completion iff $C(e)=1$ 


\section{Example Boolean trixel edge filters modeling basic perceptual criteria}

\begin{tabular}{|c|l|l|}
\hline \multirow{4}{*}{$\begin{array}{c}\text { Type } \\
\text { Spatial } \\
\text { proximity }\end{array}$} & Name & \multicolumn{1}{|c|}{ Description } \\
\cline { 2 - 3 } & SooLong & Length > median trixel edge length \\
\cline { 2 - 3 } & Longest & Shortest edge of at least one flanking trixel \\
\hline \multirow{3}{*}{$\begin{array}{l}\text { Spectral } \\
\text { proximity }\end{array}$} & NearColor & $\begin{array}{l}\text { Scaled intensities of flanking trixels or inner } \\
\text { product of unit RGB vectors of flanking trixels is } \\
\text { within threshold distance of } 1 \text { for color images }\end{array}$ \\
\hline \multirow{3}{*}{$\begin{array}{l}\text { Contour- } \\
\text { continuity }\end{array}$} & Canny & Coincides with a contour chain edge \\
\cline { 2 - 3 } & EndLink1 & $\begin{array}{l}\text { Smoothly connects end point of a contour to the } \\
\text { interior of another contour (transversality) }\end{array}$ \\
\cline { 2 - 3 } & EndLink2 & Connects two contour endpoints \\
\cline { 2 - 3 } & Junction & $\begin{array}{l}\text { At least one flanking trixel has all its vertices on } \\
\text { different contours }\end{array}$ \\
\hline
\end{tabular}

C = TooLong $\mid \sim($ Canny $\mid$ EndLink1 | EndLink2 | (Junction \& Shortest)) 


\section{Modeling Perceptual Organization}

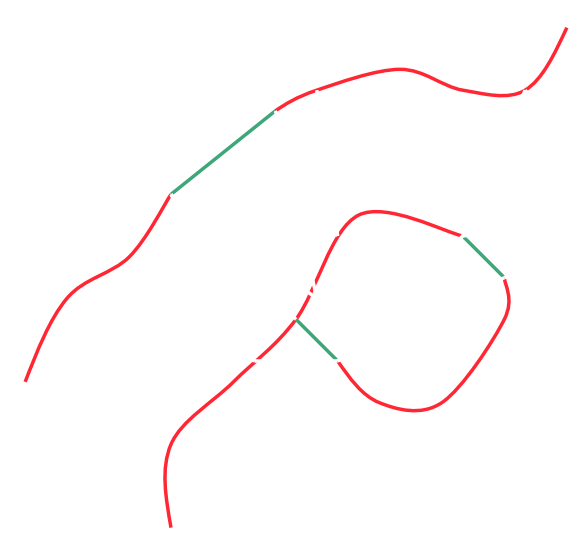

Trixel edges that are assigned the value 1 (closed) are retained as contour continuations

Every time a trixel edge is retained, a contour continuation is achieved

Every time a trixel edge is deleted, a region continuation is achieved

Thus the contour-region duality of segmentation is effected by the retention and deletion of trixel edges 


\section{VISTA: From Pixels To Polygons}

VISTA: Vectorized Image Segmentation via Trixel Agglomeration L. Prasad, A. Skourikhine, Pattern Recognition, 2006

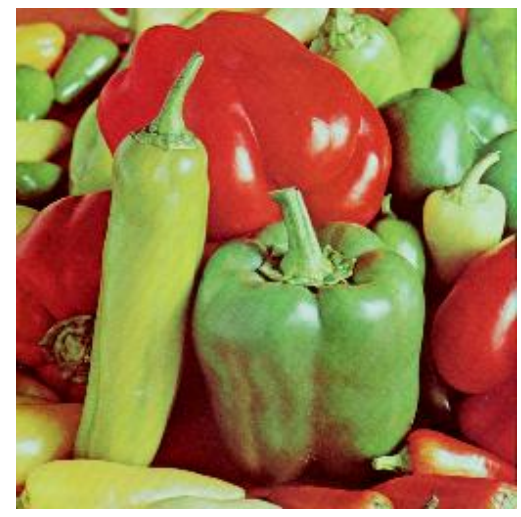

Raster image of pixels

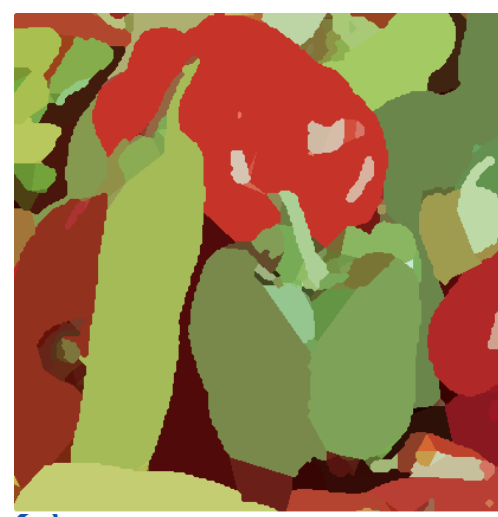

Edge

Detection

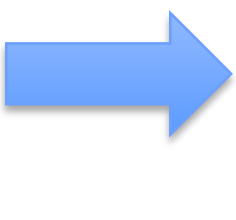

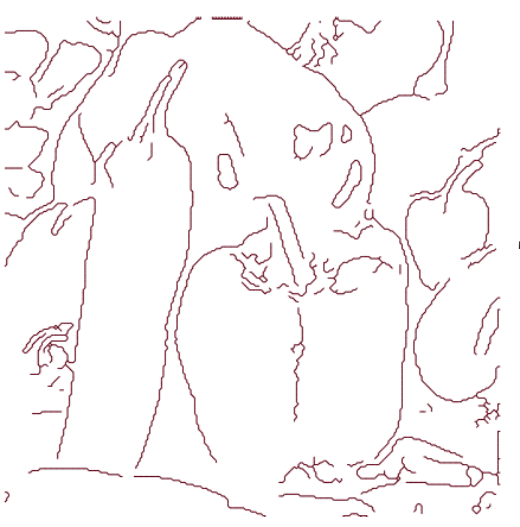

Delaunay

Triangulation

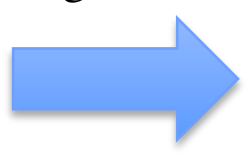

Perceptual

Grouping

Of Triangles

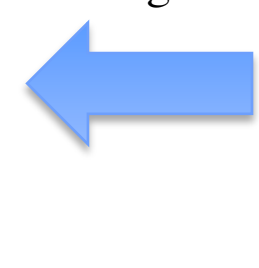

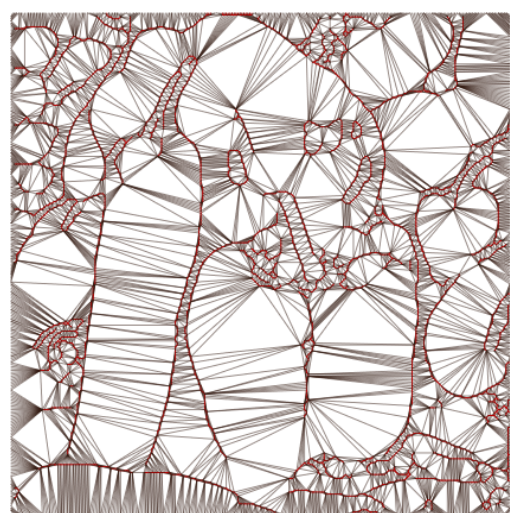

Color

Sampling

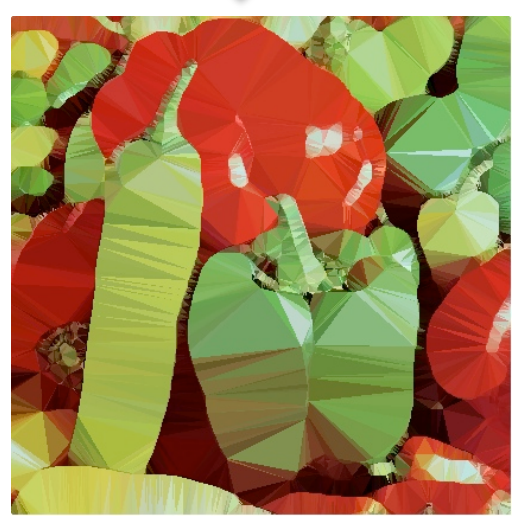




\section{Reconstruction by VISTA}
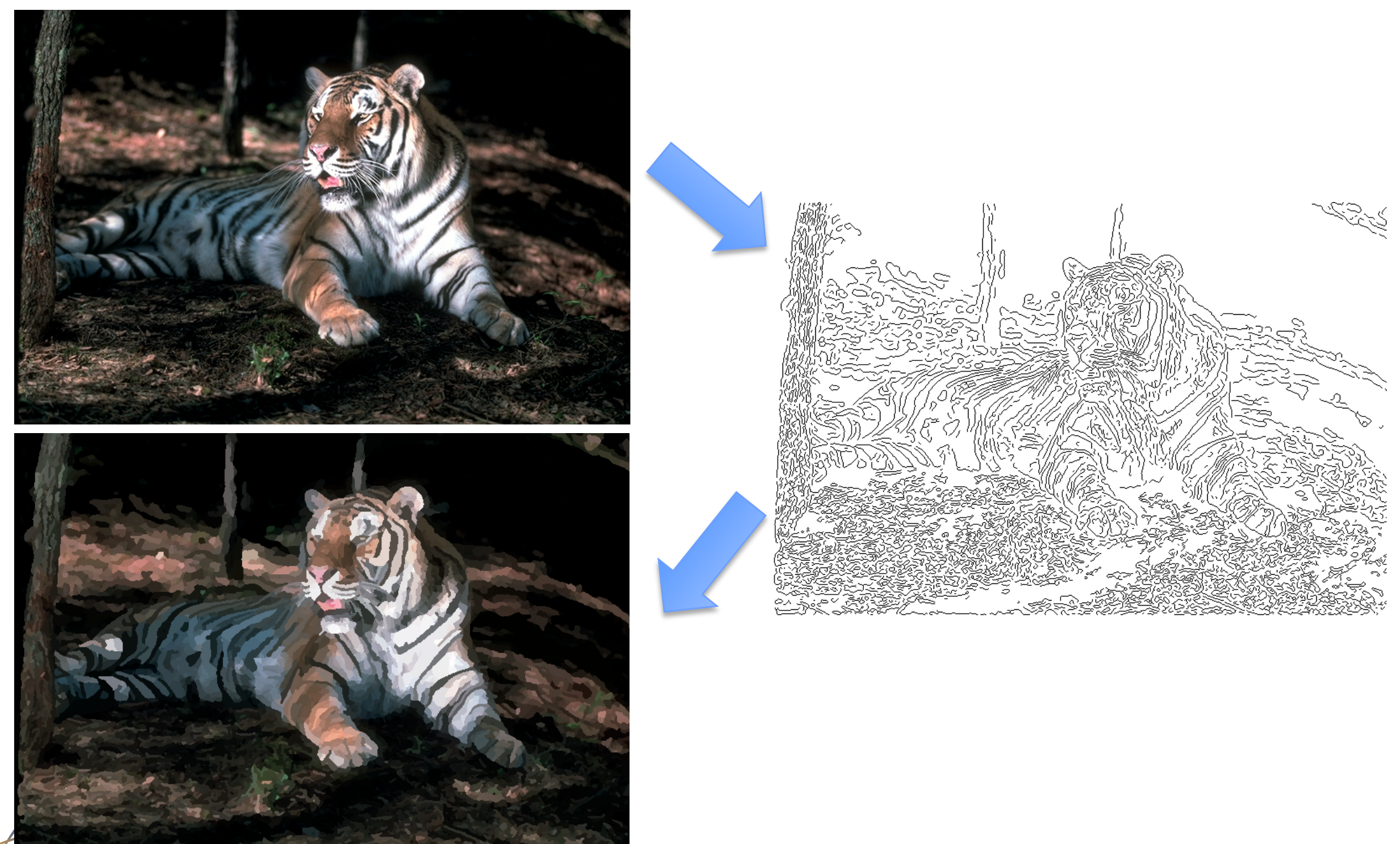

- Lośs Alamos 


\section{Aerial image segmentation and detail}

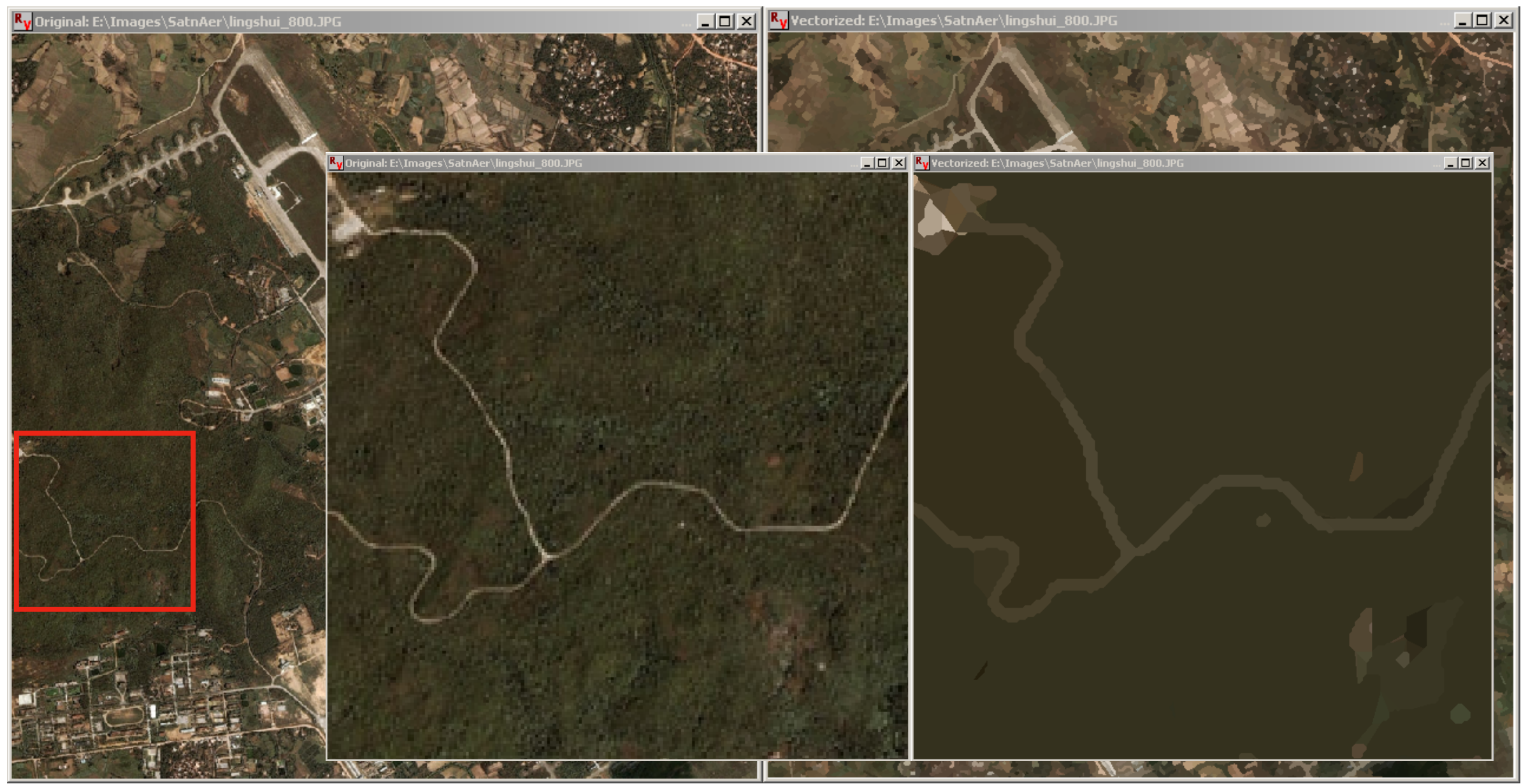




\section{Hierarchical Segmentation}

- VISTA typically provides a fine-scale segmentation

- Its polygons conform to image contours

- So, merging polygons preserves image contour fidelity

- Merge polygons in a perceptually meaningful manner

- Merge rules predicated on ecological statistics of polygon attributes: e.g., distributions of area, color, smoothness

- Progressively reduce number of regions while preserving contour saliency and spectral homogeneity

- Determine thresholds/parameters from local and global statistics of polygon attributes 


\section{Hierarchical Image Segmentation by Polygon Grouping}

Proc. IEEE CVPR Workshop on Perceptual Organization in Computer Vision 2008, AK

\section{Basic Algorithm}

1. Compute polygons from fine scale edges using the VISTA algorithm

2. Compute optimal histograms of polygon attributes: areas, color/intensity differences, orientation, etc. (H. Shimazaki, S. Shinomoto, Neural Computation 19, 2007)

3. Use modes of histograms as clusterings of polygons in attribute space

4. Merge polygons with areas less than or equal to the area of dominant granularity with adjacent polygon of minimal distance in attribute space ( e.g., color, intensity, edge saliency cost)

5. Repeat steps 2 through 4 until number of polygons converges. 


\section{Hierarchical multiscale image segmentation: example}

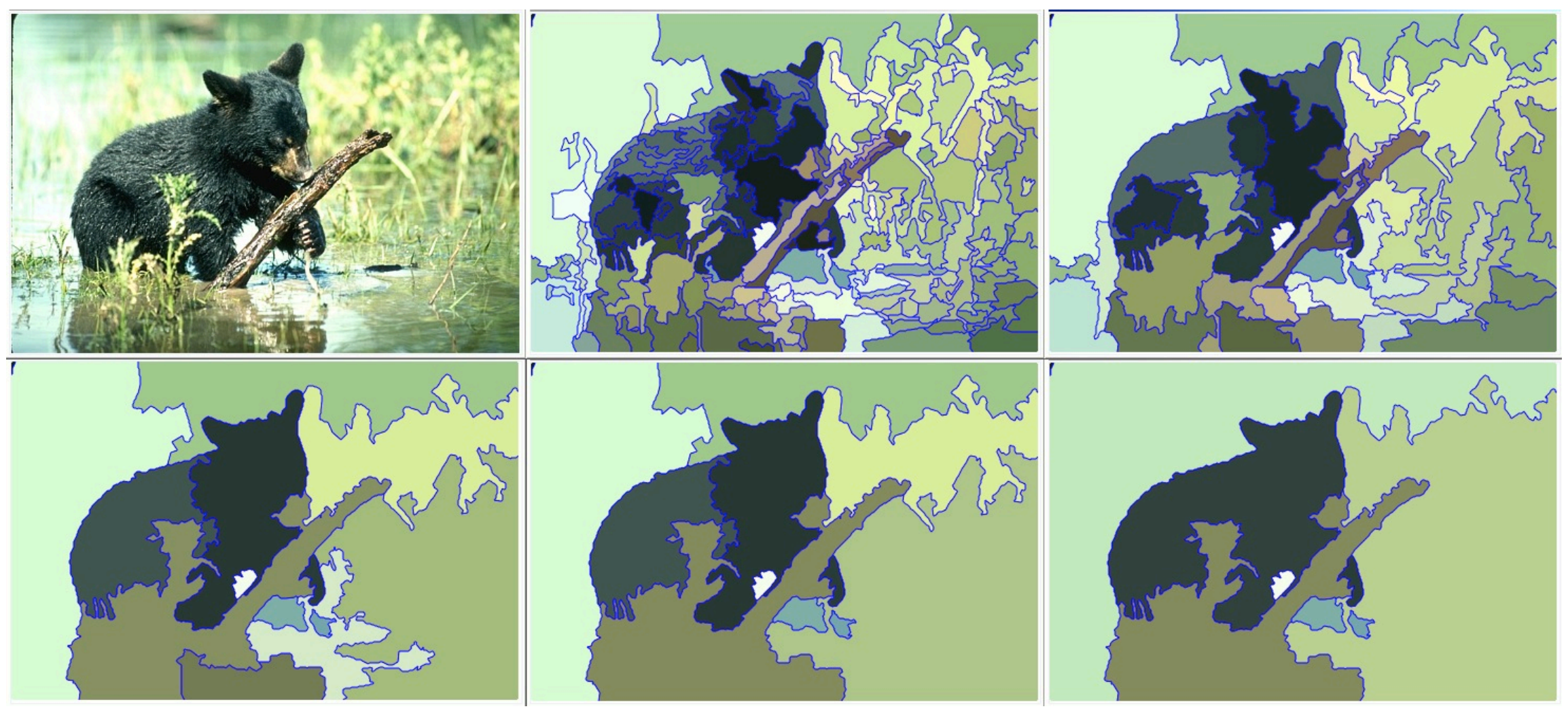

Emergence of fine to coarse features by successive polygon merging 
Hierarchitect: a 2-stage algorithm to build the segmentation hierarchy

Stage 1 constructs polygonal images $\tilde{I}$ that approximate the original raster image $I$ while being maximally smooth in region interiors and minimizing number of regions (interface boundaries).

In spirit, it seeks to minimize the Mumford-Shah functional:

( $\Omega$ : image domain, $\Gamma$ : image contours) STAGE 1:

$$
F(\tilde{I}, \Gamma)=\int_{\Omega-\Gamma}(\tilde{I}-I)^{2} d x d y+\alpha \int_{\Omega-\Gamma}|\nabla \tilde{I}|^{2} d x d y+\beta \int_{\Gamma} d s
$$

Start with a fine-scale segmentation of image $I$ provided by VISTA as level 1 , $L^{1}$, of hierarchy

- If a polygon $P$ in $L^{i}$ is deemed a grain*, then merge with those adjacent neighbors having non-smooth common boundary with $P$ that are closest in color to $P$.

( ${ }^{*}$ If $A$ is the area distribution of polygons in $L^{i}$, then a polygon $P$ is a grain if $\operatorname{area}(P) \leq \mu(\mathcal{A})+\sigma(\not A)$.)

Denoting by $P \cap Q$ the common boundary between $P$ and $Q$, we define the 'wiggliness' of $P \cap Q$ by $W(P \cap Q)=1-\frac{\ell_{S}(P \cap Q)}{\ell(P \cap Q)} \quad$ where $\ell$ and $\ell_{S}$ denote the length and the smoothed length of $P \cap Q$.W captures the degree of nonsmoothness of polygon boundary interfaces.

Iterate until $\# L^{i}=\# L^{i-1}$ or the median no. of $L^{l}$ polygons in polygons of $L^{i}>\# L^{i}$. 
Hierarchitect: a 2-stage algorithm to build the segmentation hierarchy

At the end of Stage 1 each polygon $P$ in $L^{i}$ is, on average, composed of several

$L^{1}$ polygons. Accordingly, its spectral composition is represented by an area-weighted distribution $D(P)$ of colors of its constituent $L^{1}$ polygons. spectral distance between polygons $P \& Q$ is given by:

$D(I)$ is the RGB distribution of all $L^{1}$ polygons of image $I$

$$
d C(P, Q)=\sum_{k=R, G, B} \sum_{j=1}^{256} \frac{\left(D_{j}^{k}(P)-D_{j}^{k}(Q)\right)^{2}}{D_{j}^{k}(I)}
$$

external disparity of a polygon $P$ in $L^{i}$ - a measure of dissimilarity with respect

to its neighbors: $\operatorname{DispE}(P)=\min _{Q \in N b d(P)}\{d C(P, Q)\}$

internal disparity is a measure of heterogeneity of the polygon $P$ in terms of the

$L^{i-1}$ polygons constituting it: $\operatorname{DispI}(P)=\underset{Q \subseteq P}{\operatorname{median}}\{d C(P, Q)\}$

An interface boundary $B$ in $L^{i}$ is deemed salient if

$$
\left(\ell(B) \geq \underset{P \in L^{i}}{\operatorname{median}}\left\{\max _{Q \in N b d(P)}\{\ell(P \cap Q)\}\right\}\right) \wedge\left(W(B) \leq \operatorname{median}_{P \in L^{i}}\left\{\min _{Q \in N b d(P)}\{W(P \cap Q)\}\right\}\right)
$$

$B=P \cap Q \quad$ is deemed a pseudoboundary if it is not salient and if either

$$
W(B)=W(P \cup Q) \leq \min (W(P), W(Q)) \text { or } W(P \cap Q) \geq \max \left\{W\left(B_{j}\right)\right\}
$$

$$
B_{j} \in L^{i}
$$

where $P \cup Q$ denotes the polygon that is the union of the polygons $P \& Q$. 
Hierarchitect: a 2-stage algorithm to build the segmentation hierarchy

Stage 2 seeks to merge polygons with similar spectral distributions while preserving salient boundaries between them. This stage produces features that are prominent objects or coherent backgrounds that contextually relate finer features within them

STAGE 2:

A polygon $P$ may merge with one or more of its neighbors $Q$ if all three following conditions are satisfied:

- Its external disparity is less than or equal to the median of the external disparities of all polygons at the current level, or its internal disparity is greater than its external disparity: $\quad\left(\operatorname{DispE}(P) \leq \operatorname{median}_{P^{\prime} \in L^{\prime}}\left\{\operatorname{DispE}\left(P^{\prime}\right)\right\}\right)(\operatorname{DispI}(P)>\operatorname{DispE}(P))$

- The spectral distance of its qualifying merge neighbor $Q$ is equal to the external disparity of the polygon (i.e., the neighbor is the least dissimilar): $d C(P, Q)=\operatorname{DispE}(P)$

- The boundary interface with a qualifying merge neighbor is a pseudoboundary:

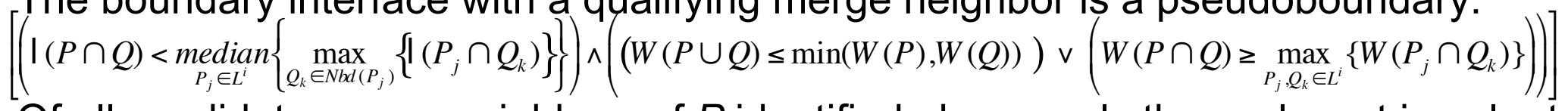

- Of all candidate merge neighbors of $P$ identified above, only those closest in color to $P$ are allowed to merge with it: $\quad\|\mu(D(P))-\mu(D(Q))\| \leq \min _{Q^{\prime} \in N b d(P)}\left\{\left|\mu(D(P))-\mu\left(D\left(Q^{\prime}\right)\right)\right|\right\}$

where $\mu(D(P))$ is the weighted average of the colors of the $L^{1}$ polygons constituting $P$.

- Iterating these steps produces new levels until no new polygonal mergers are possible. 
Hierarchical Segmentation: satellite imagery

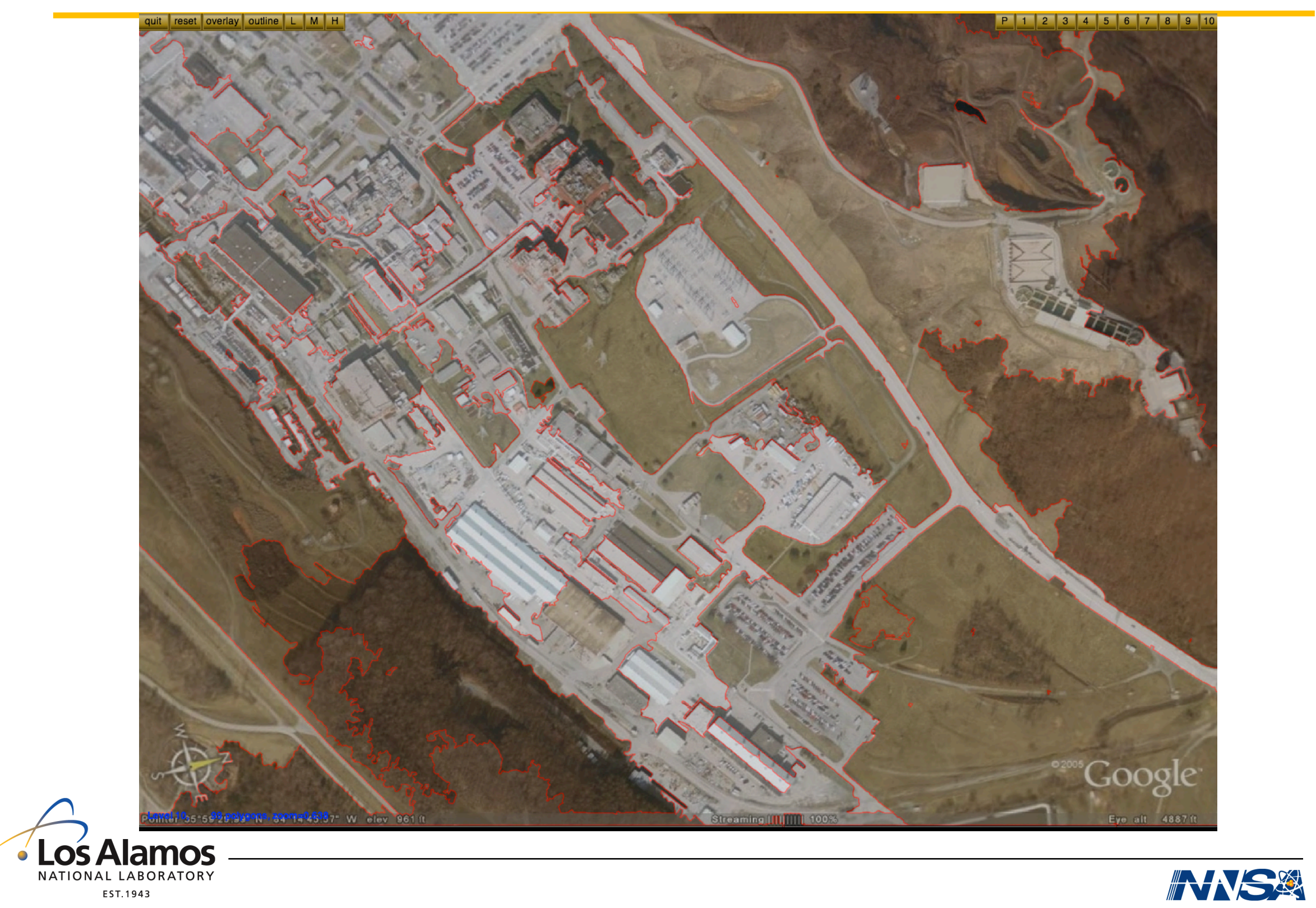


Comparison: RADIUS vs Multiscale Normalized Cut and Mean Shift

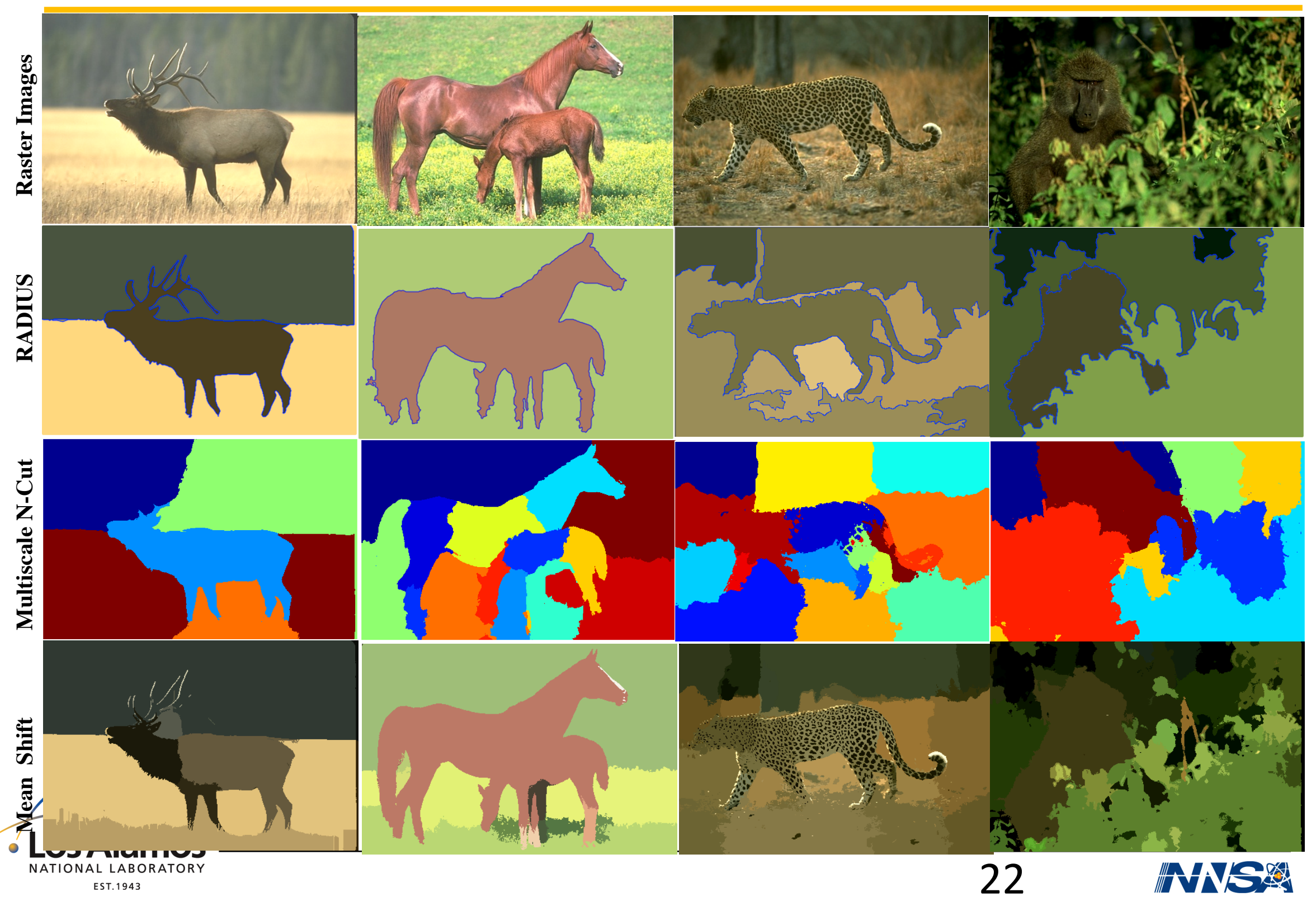




\section{Hierarchitect vs. Multiscale Normalized Cuts and Meanshift}

\begin{tabular}{|c|c|c|c|c|c|c|}
\hline \multirow{2}{*}{ Image } & \multicolumn{2}{|c|}{ Our Method } & \multicolumn{2}{c|}{ Multiscale Ncuts } & \multicolumn{2}{c|}{ Mean Shift } \\
\cline { 2 - 7 } & $\mathrm{T}$ & $\mathrm{N}$ & $\mathrm{T}$ & $\mathrm{N}$ & $\mathrm{T}$ & $\mathrm{N}$ \\
\hline Elk & 0.30 & 3 & 41.9 & 5 & 15.4 & 65 \\
\hline horse & 0.55 & 2 & 105.6 & 13 & 15.4 & 121 \\
\hline leopard & 0.40 & 11 & 156 & 17 & 47.6 & 1952 \\
\hline baboon & 0.34 & 5 & 69.2 & 7 & 15.9 & 371 \\
\hline $\begin{array}{c}\text { Comparison of run times (T) in seconds and the number of segmented regions (N) } \\
\text { obtained by our method, the multiscale normalized cuts method, and the mean shift } \\
\text { method for the eight images in previous slide. }\end{array}$ \\
\hline
\end{tabular}




\section{Perceptual Quality:}

\section{Comparison with Berkeley Segmentation Dataset benchmark}

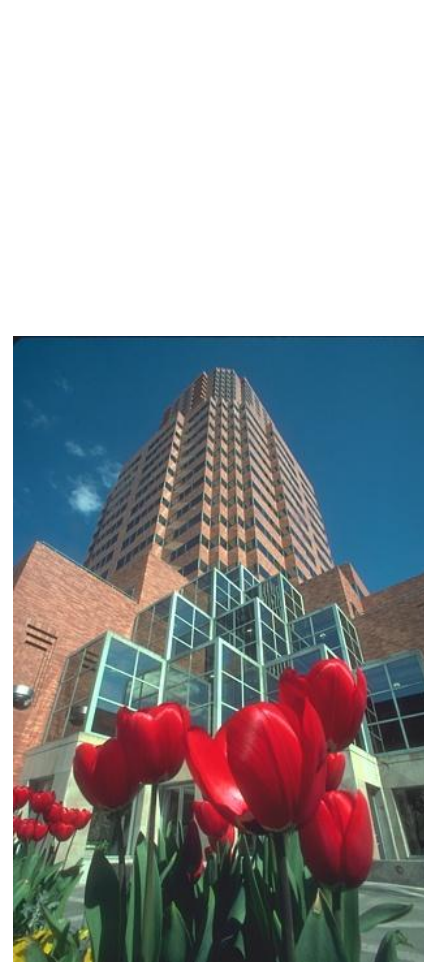

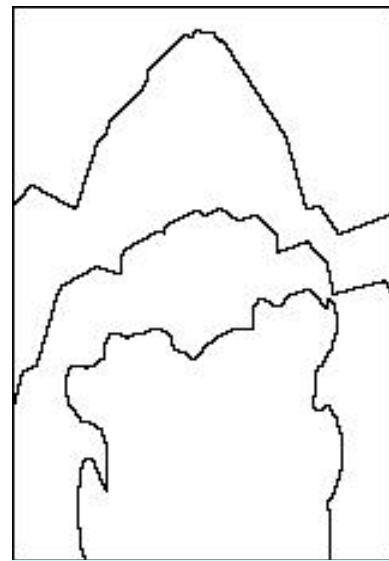

User \#1116

4 Segments

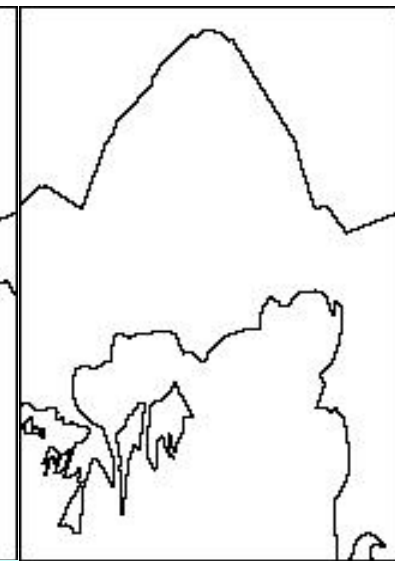

User \#1105 12 Segments

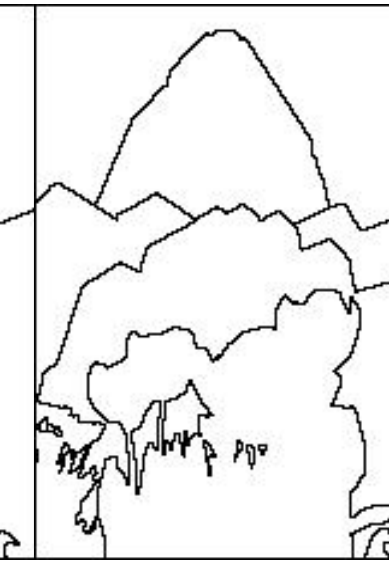

User \#1115

27 Segments

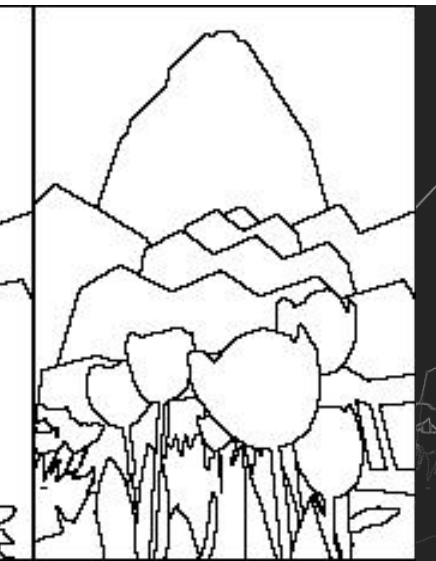

User \#1127

67 Segments
BSDS persistence map

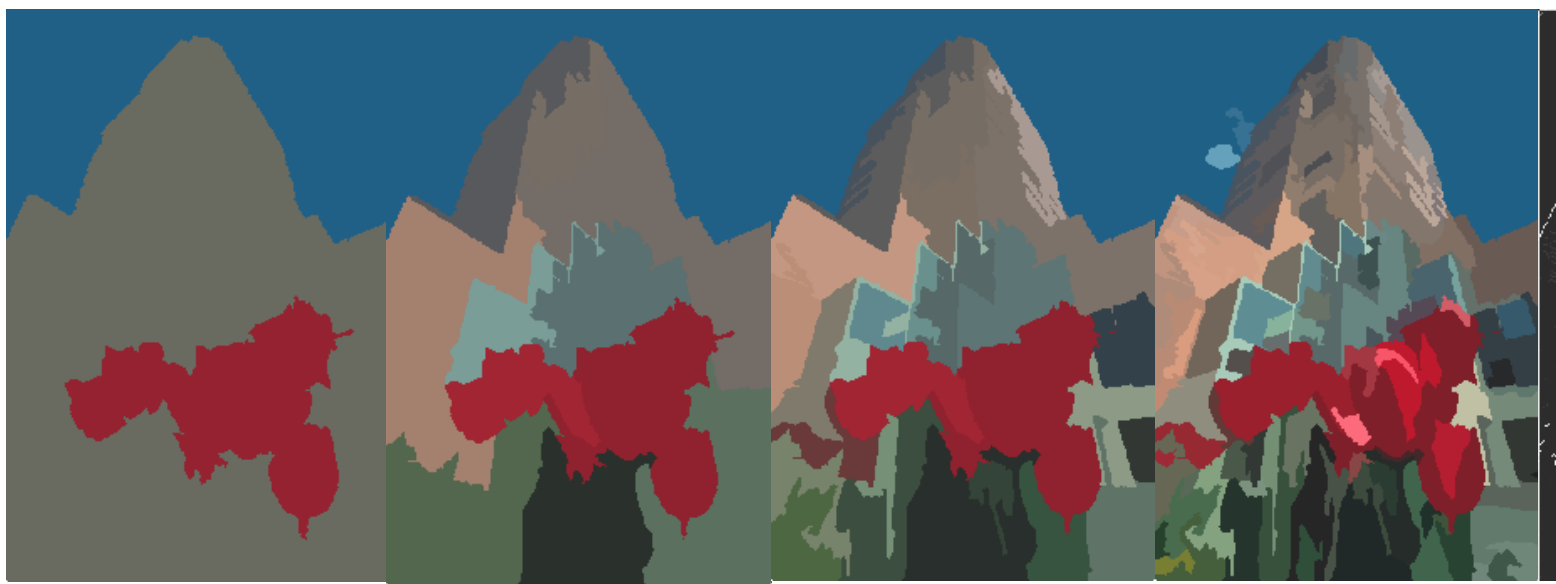

Hierarchitect Level 6 Hierarchitect Level 5 3 Segments
Hierarchitect Level 4 29 Segments
Hierarchitect Level 3 116 Segments
Hierarchitect persistence map 


\section{Benchmarking RADIUS and Quantitative Comparison}

Berkeley Image segmentation dataset: 300 images representing a wide range of image complexity. All have multiple ground truth segmentations by human subjects.

Rand index: Measures the similarity between two segmentations of an image: If $\boldsymbol{S}=\left\{\boldsymbol{s}_{i}\right\}_{i=1 \ldots N}$ is a set of elements, and $\mathcal{C}=\left\{\mathbf{C}_{1}, \ldots \mathbf{C}_{m}\right\}$ and $\boldsymbol{D}=\left\{\mathbf{D}_{1}, \ldots, \mathbf{D}_{n}\right\}$ are two clusterings of the elements of $S$, then the Rand index of the two clusterings is given by:

$\mathbf{R}(\mathbf{C}, \mathbf{D})=(\#\{$ element pairs of $\boldsymbol{s}$ belonging to same cluster in both $\mathcal{e}$ and $\boldsymbol{D}\}+$ $\#\{$ element pairs of $S$ not belonging to same cluster in either $e$ or $D\}) /{ }^{N} C_{2}$

The mean Rand index of a test clustering with a set of reference clusterings is its probabilistic rand index (PRI).

\begin{tabular}{|c|c|c|c|c|c|c|c|c|c|c|c|}
\hline Algorithm & Meanshift & $\begin{array}{l}\text { Normalized } \\
\text { Cuts }\end{array}$ & Edge Flow & \begin{tabular}{|l}
$\begin{array}{l}\text { Graph-based } \\
\text { Segmentation }\end{array}$ \\
Sols
\end{tabular} & \begin{tabular}{|l|} 
Multiscale \\
Normalized Cu
\end{tabular} & \begin{tabular}{|l} 
Normalized Tree \\
Partitioning
\end{tabular} & \begin{tabular}{|l|} 
Saliency Total \\
Variation
\end{tabular} & \begin{tabular}{|l|} 
Compressive \\
Texture Merging
\end{tabular} & $\begin{array}{l}\text { Texture } \\
\text { Boundary } \\
\text { Evondry }\end{array}$ & RADIUS & $\begin{array}{l}\text { Human } \\
\text { Segmentations }\end{array}$ \\
\hline $\begin{array}{l}\text { PRI score } \\
(\max =1)\end{array}$ & 0.79 & 0.72 & 0.77 & 0.71 & 0.76 & 0.75 & 0.78 & 0.77 & 0.80 & 0.82 & 0.87 \\
\hline
\end{tabular}




\section{Parallelization for Large Images}

P processors

$\mathrm{N}$ pixels

Each processor does N/P work.

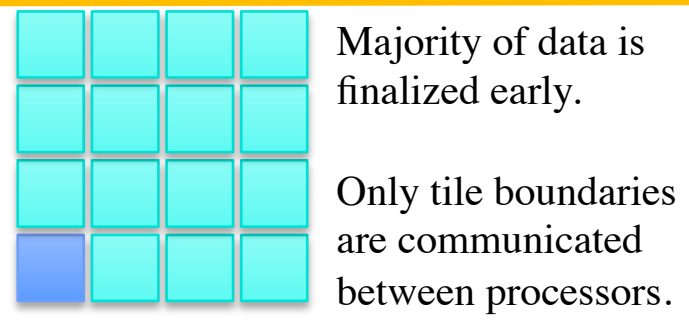

between processors.
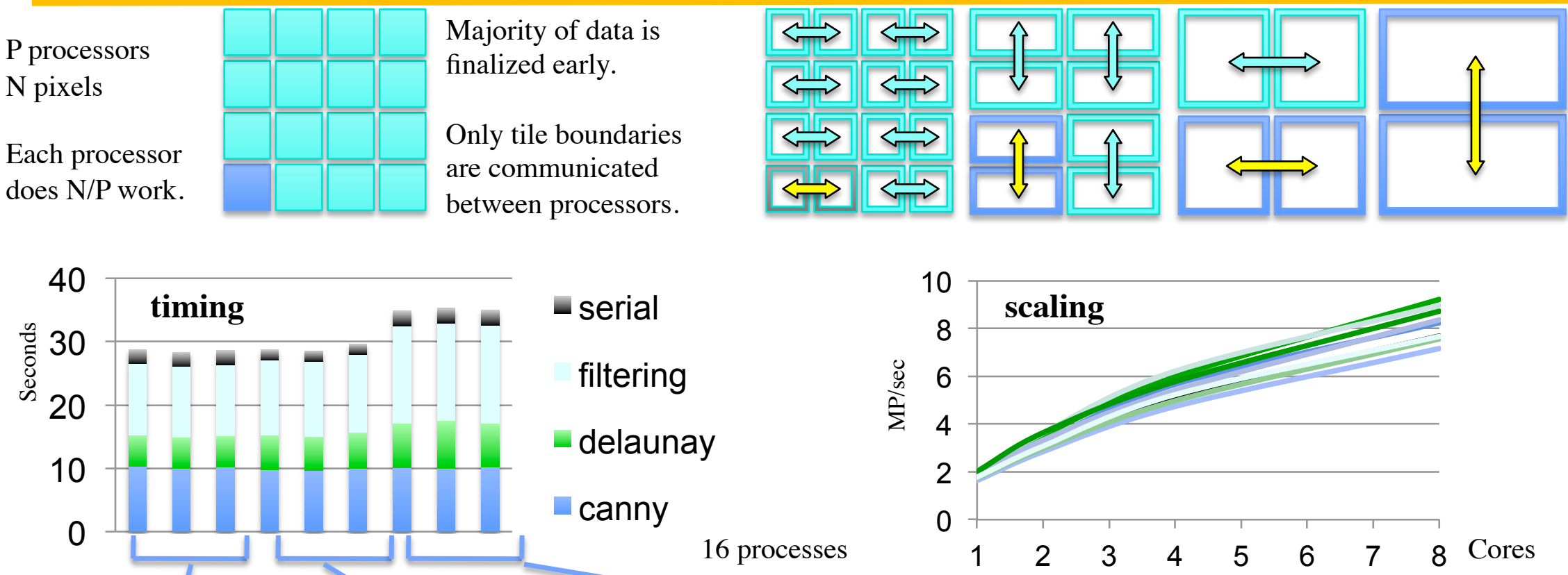


\section{Mining the hierarchy for features of interest}

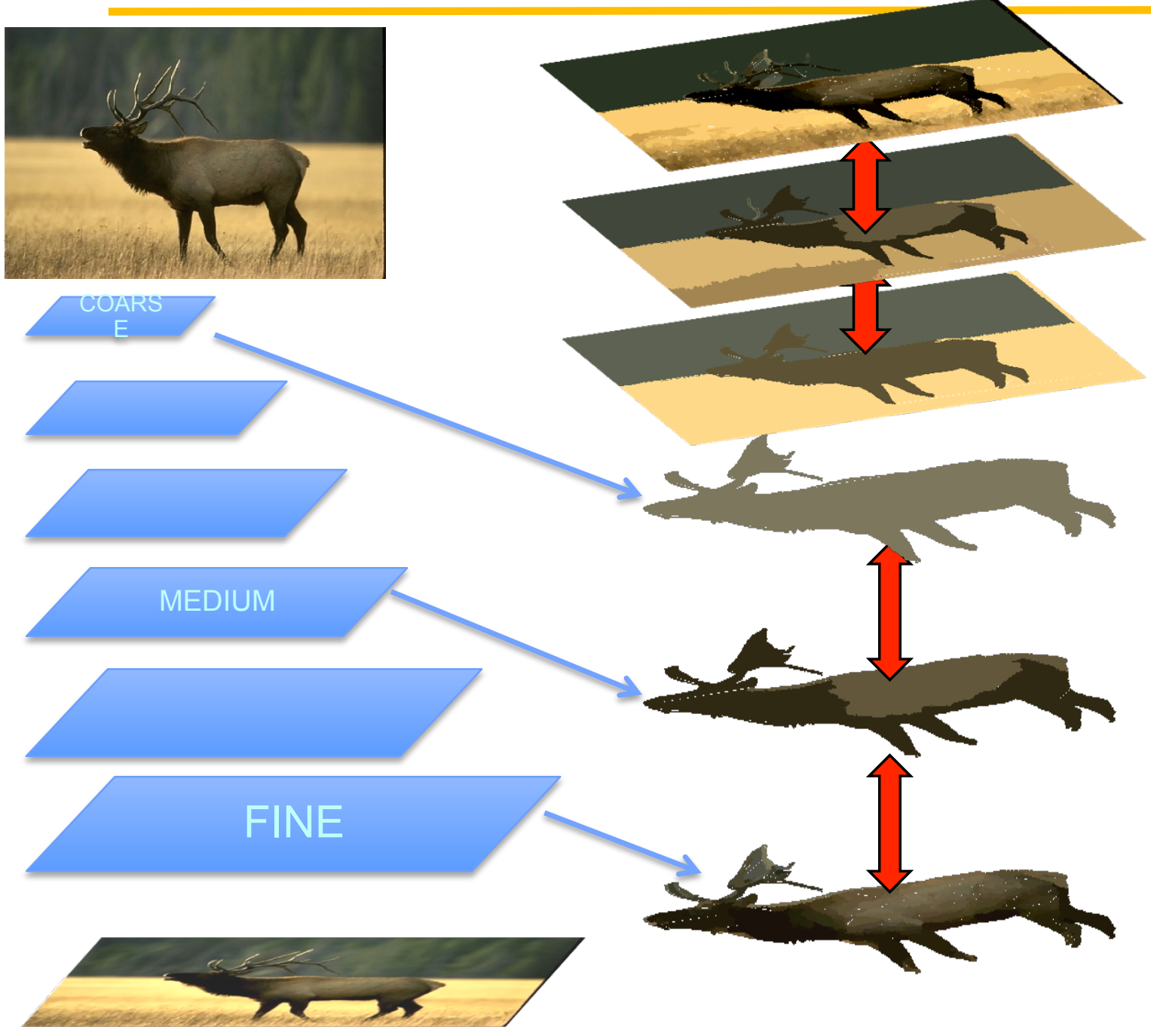

The hierarchy contains fine to coarse segmentations of features at various levels.

Detecting, extracting, and relating features of interest is the next challenge

What general principles of 'interest' should one apply?

Shape, smoothness, color, scale, context, ..., saliency, anomaly?

The hierarchy is designed to address all these aspects of images efficiently,

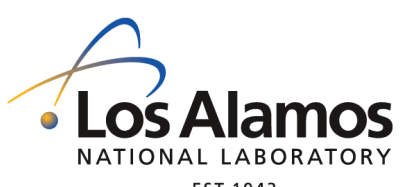


Using the Hierarchy: Adaptive multiple backgrounds for contextual saliency detection

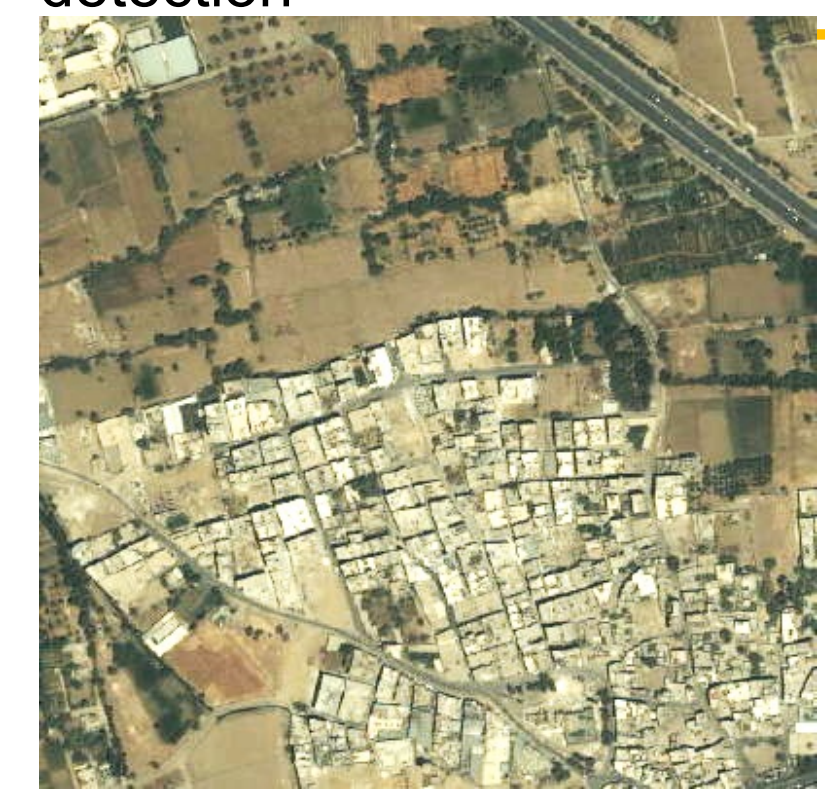

Fig.1 Raster aerial image
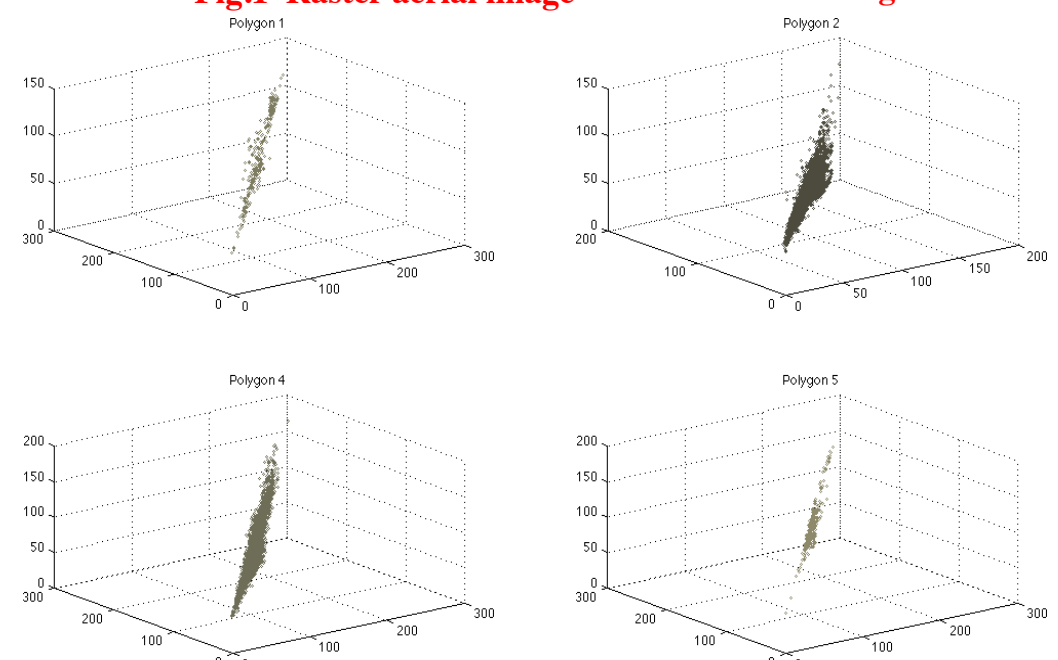

- LOŚS Álamos

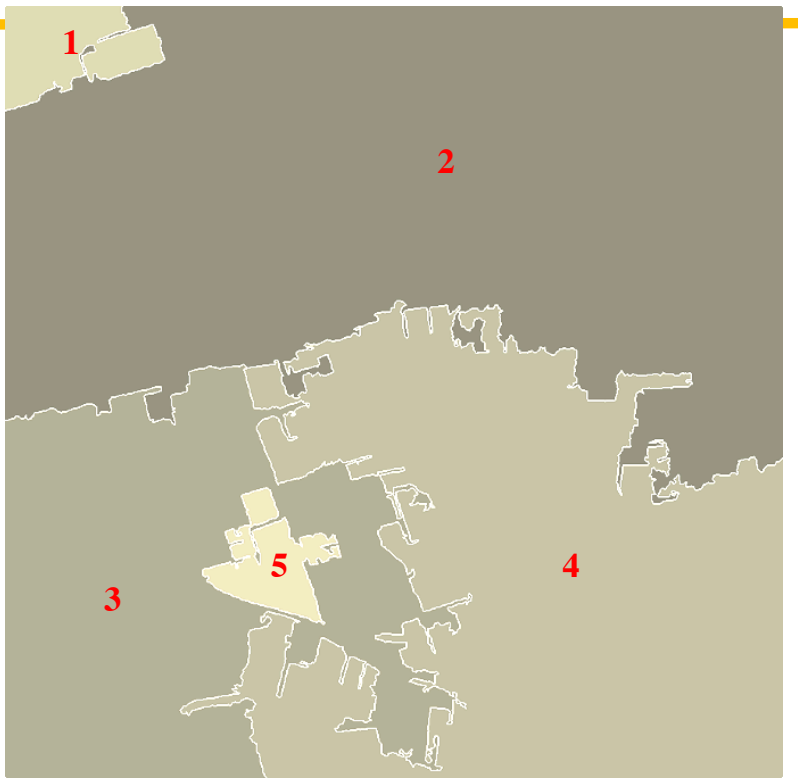

Fig. 2 Coarse-level background polygons

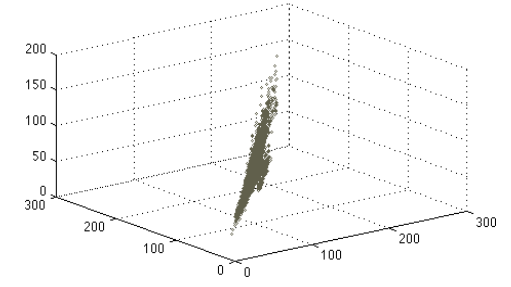

Fig. 4 Distributions of RGB values of level-1 descendents of the five background polygons

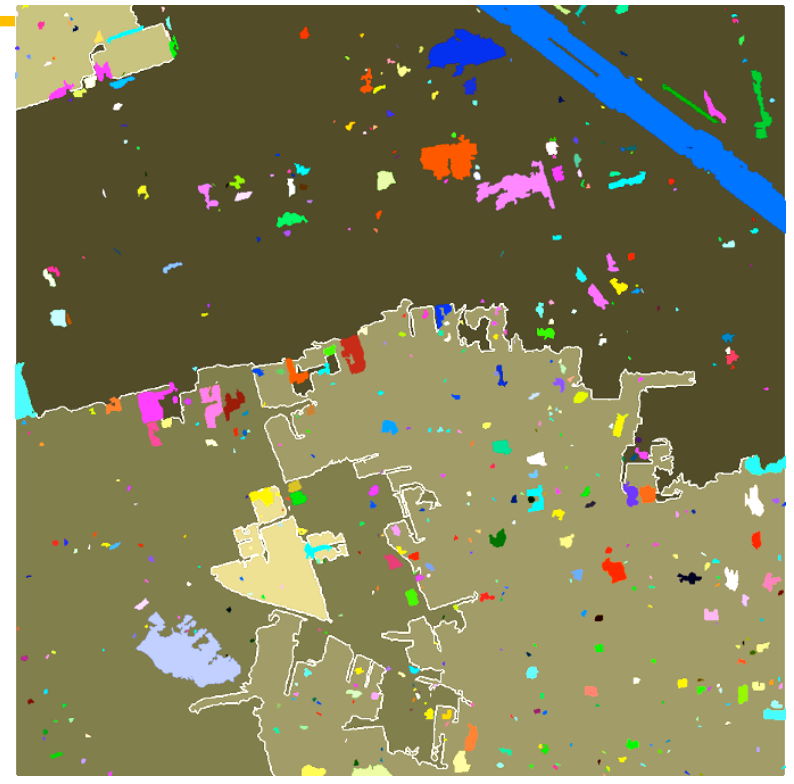

Fig. 3 Salient features detected as anomalies

Spectral distributions of level-1 descendents of background polygons are approximately ellipsoidal in nature (Fig. 4), due to spectral homogeneity of

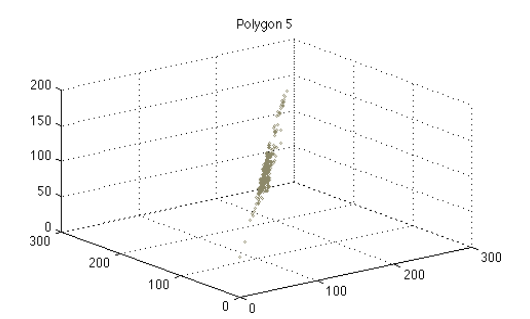

segments.

Therefore salient features may be modeled as outliers w.r.t. the Mahalanobis distance of the samples from the mean (Fig. 3) 


\section{Using the Hierarchy: Adaptive multiple backgrounds for contextual saliency detection}

Hierarchical Segmentation

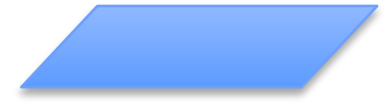

Background polygon
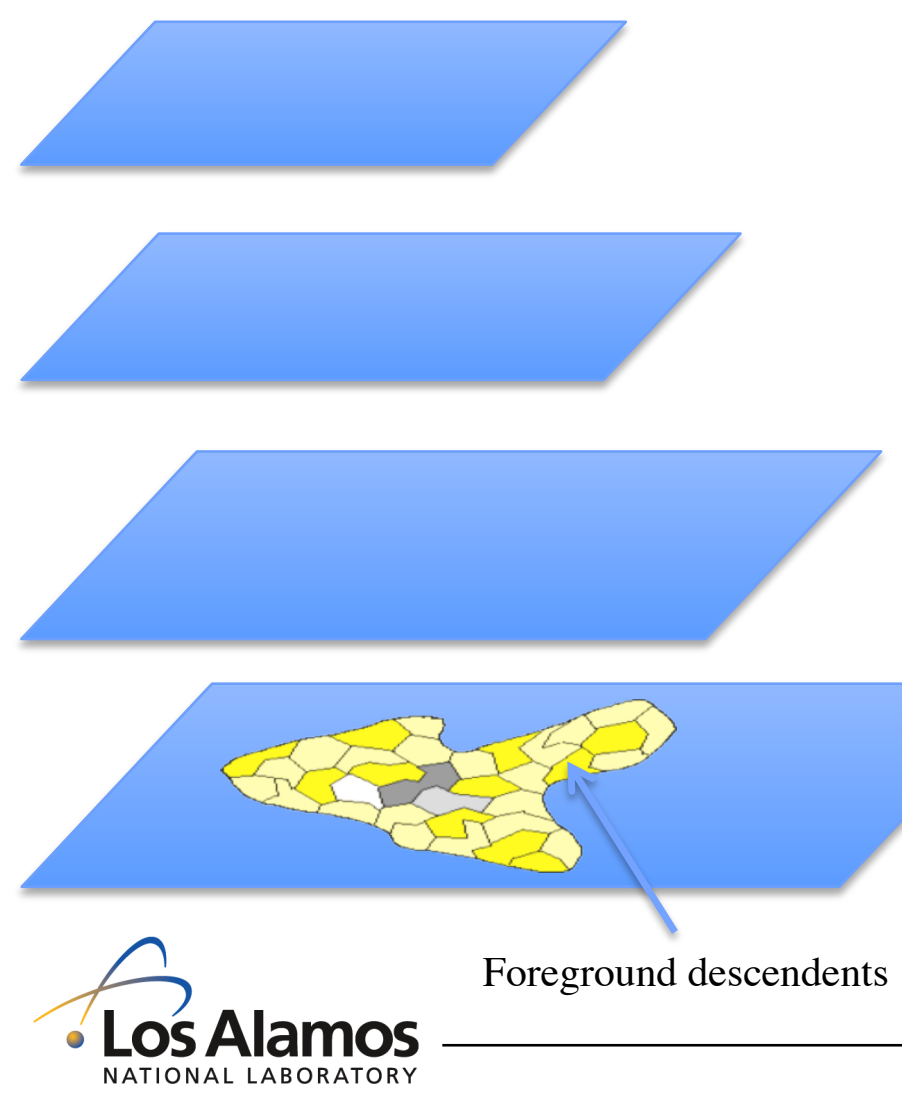

Foreground descendents

\section{Salient Feature Detection Scheme:}

1. Hierarchical segmentation of image

2. Selection of background polygon and foreground level

3. Anomaly detection on foreground descendents

4. Hierarchical grouping of anomalous polygons

Recovered feature

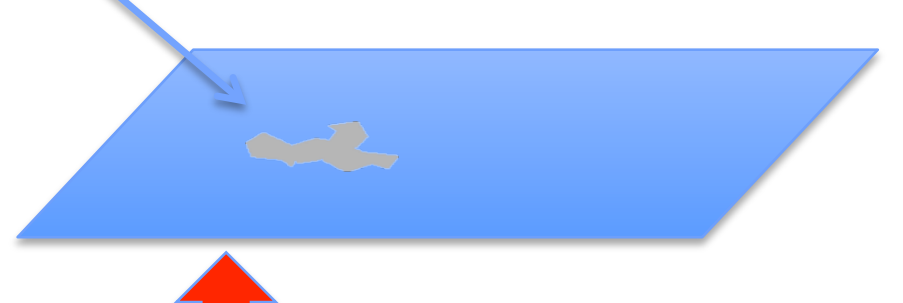

Hierarchical polygon grouping

Anomalous polygons 


\section{Segmentation by Contextual Saliency Detection}

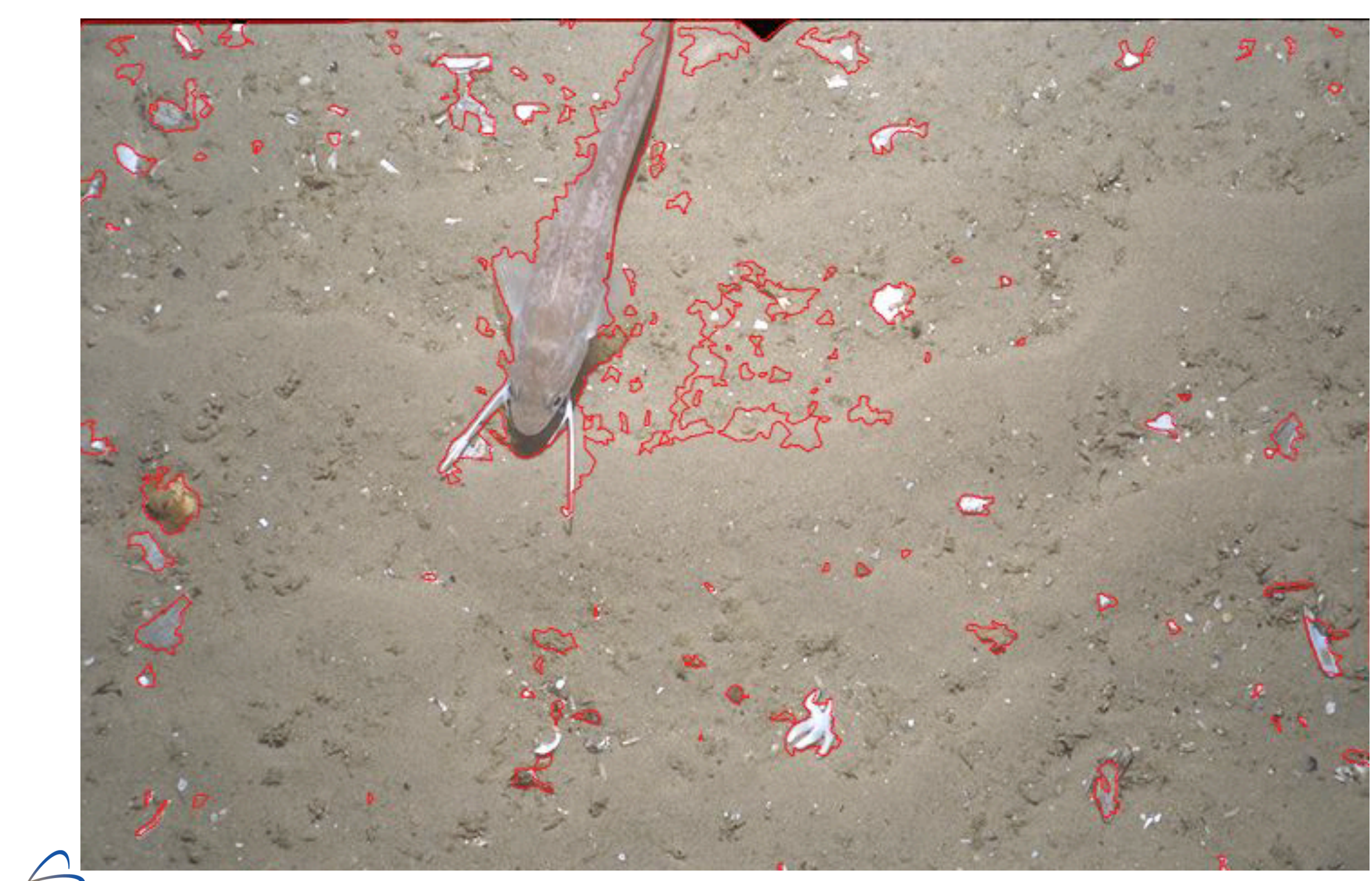




\section{Segmentation by Contextual Saliency Detection}

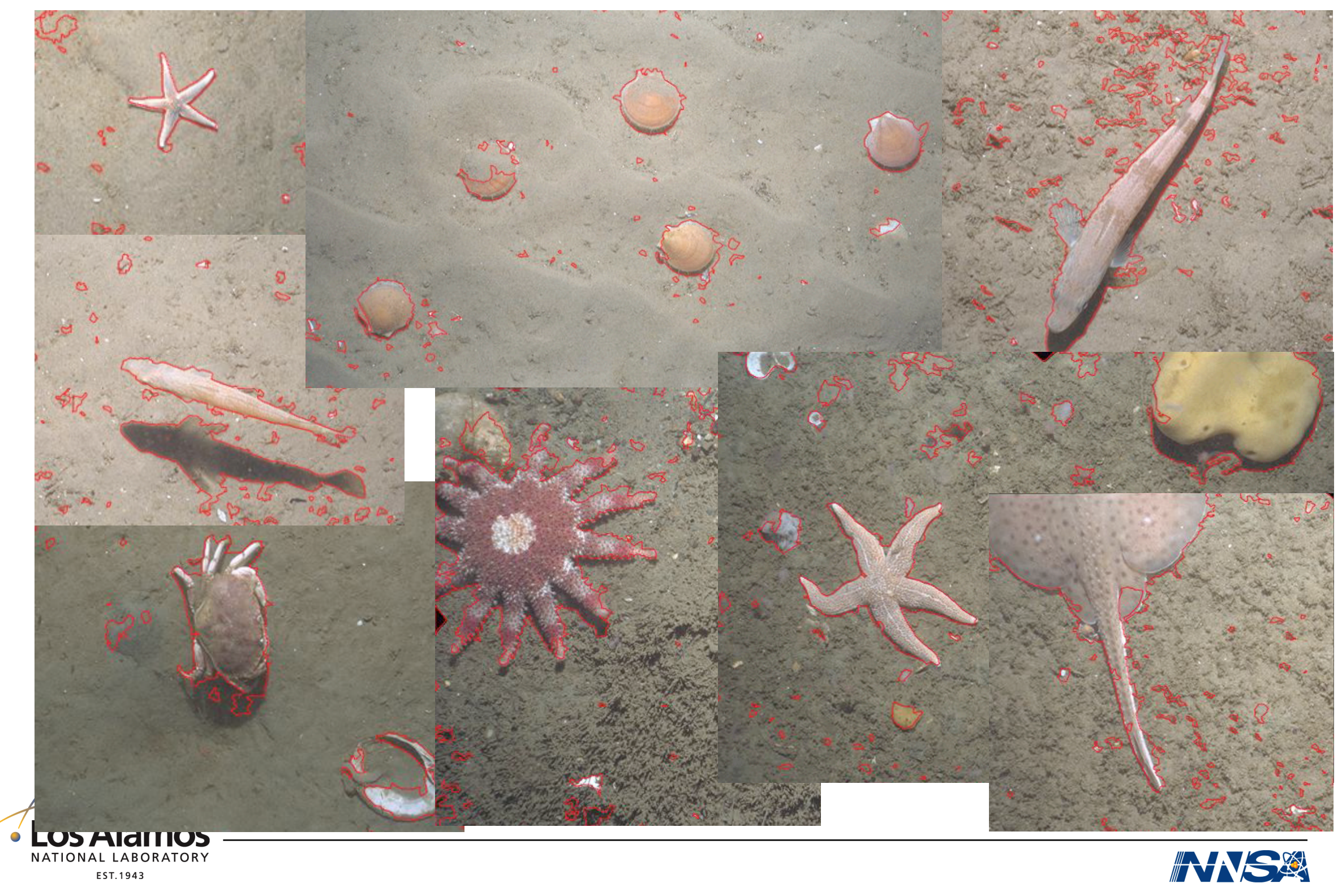




\section{Camouflage Detection and Defeat:}
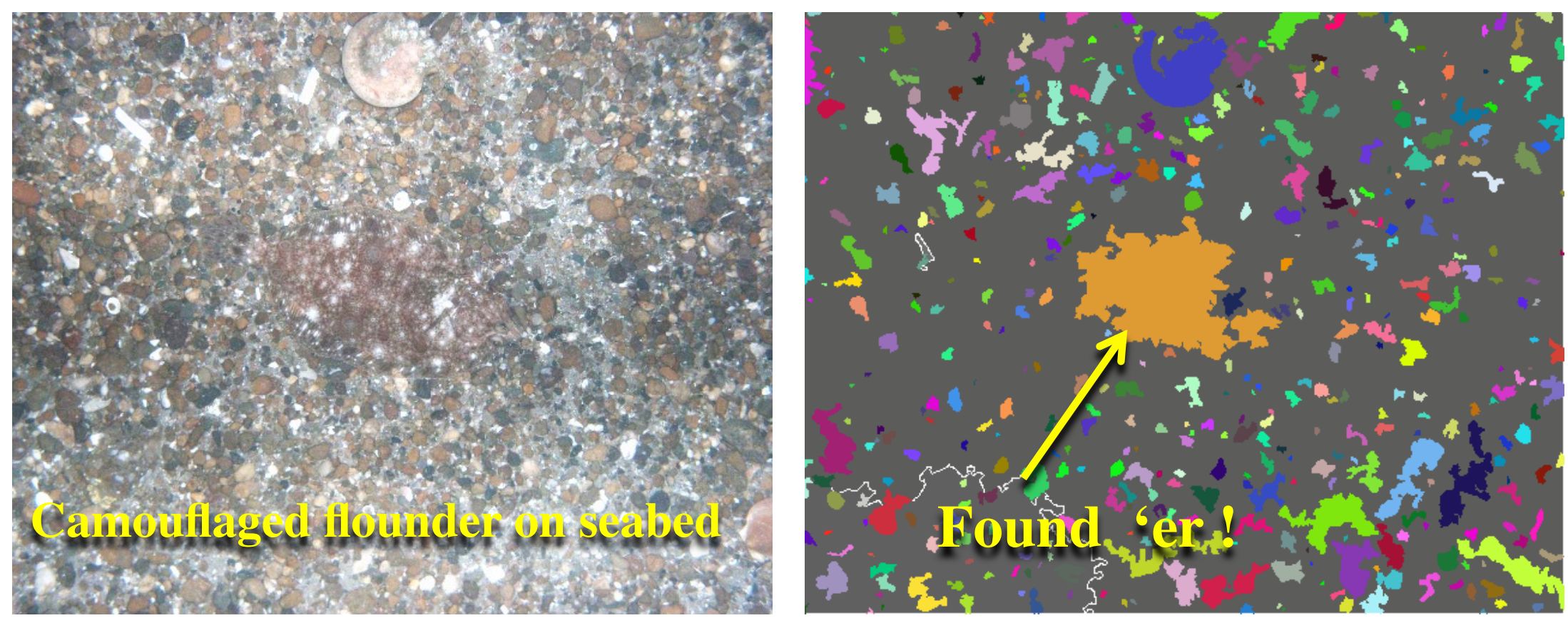


\section{Discriminating Structure from Texture}
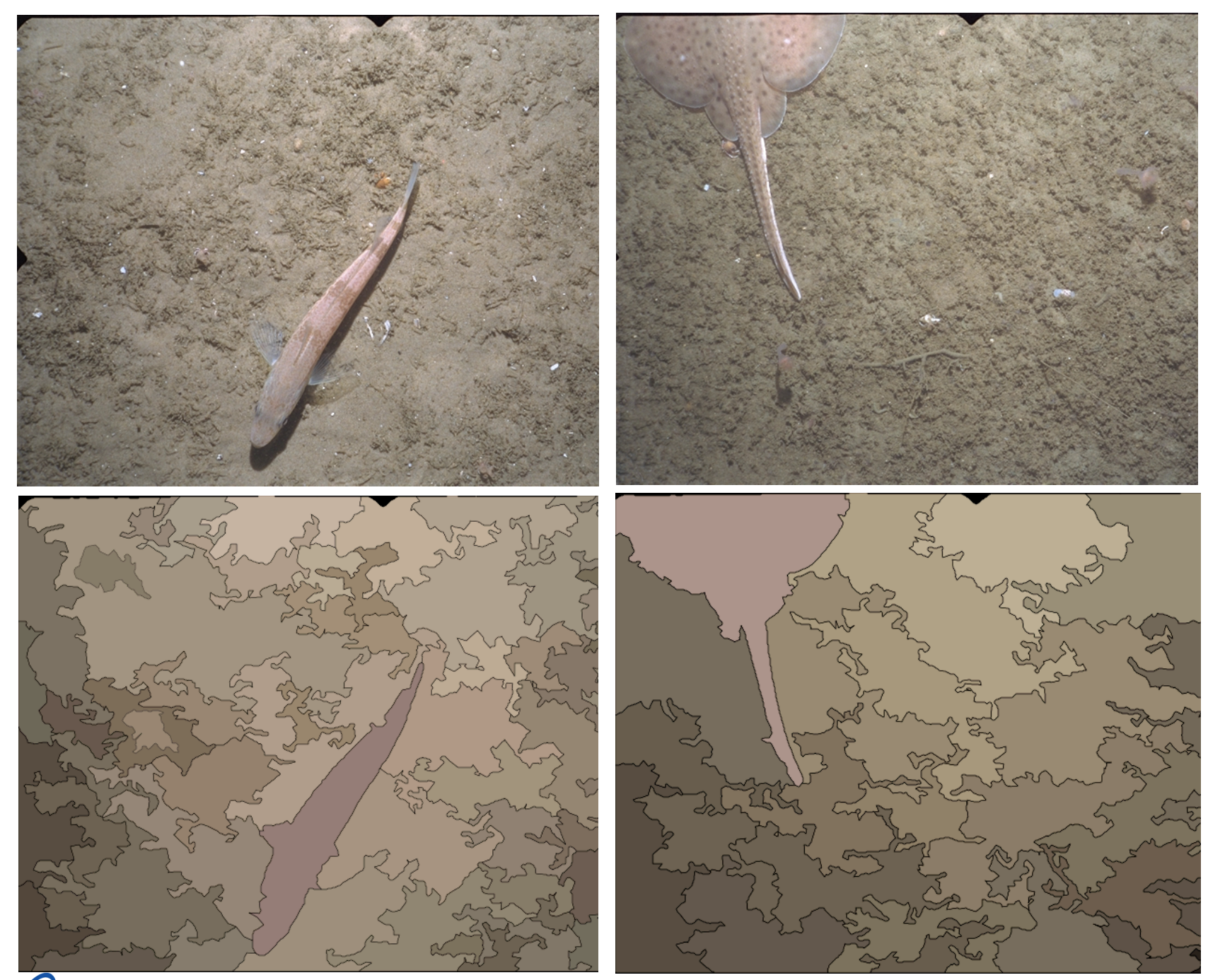

In the successive grouping of polygons, texture regions segment into polygons with high energy boundaries, whereas objects tend to have more regular boundaries.

This can be exploited as a later stage perceptual cue that can distinguish texture from structure. 


\section{Flattening texture by minimizing contour wiggle}

Define wiggle in the boundary of a polygon $A$ as $\quad w(A)=1-\frac{L_{S}(A)}{L(A)}$

For adjacent polygons $A$ and $B$

and

$$
\begin{gathered}
L(A \cup B)=L(A)+L(B)-2 L(A \cap B) \\
L_{S}(A \cup B) \leq L_{S}(A)+L_{S}(B)-2 L_{S}(A \cap B)
\end{gathered}
$$

A good approximation: $\quad w(A \cup B) \approx \frac{w(A) L(A)+w(B) L(B)-2 w(A \cap B) L(A \cap B)}{L(A)+L(B)-2 L(A \cap B)}$

Merge $A$ and $B$ iff

$w(A \cup B) \leq \min (w(A), w(B))$ 
Temperature of a curve: Blaschke-Steinhaus Theorem Detecting wiggly curves and texture

If $\Gamma$ is a compact curve in the plane then the expected value of of the number of its intersections with any straight line is given by

$$
\langle X\rangle=\frac{2|\Gamma|}{|C H(\Gamma)|}
$$

Where $\mathrm{CH}(\Gamma)$ is the convex hull of $\Gamma$.

The 'temperature' of $\Gamma$ is defined as

$$
T(\Gamma)=-\left[\ln \left(1-\frac{1}{\langle X\rangle}\right)\right]^{-1}
$$

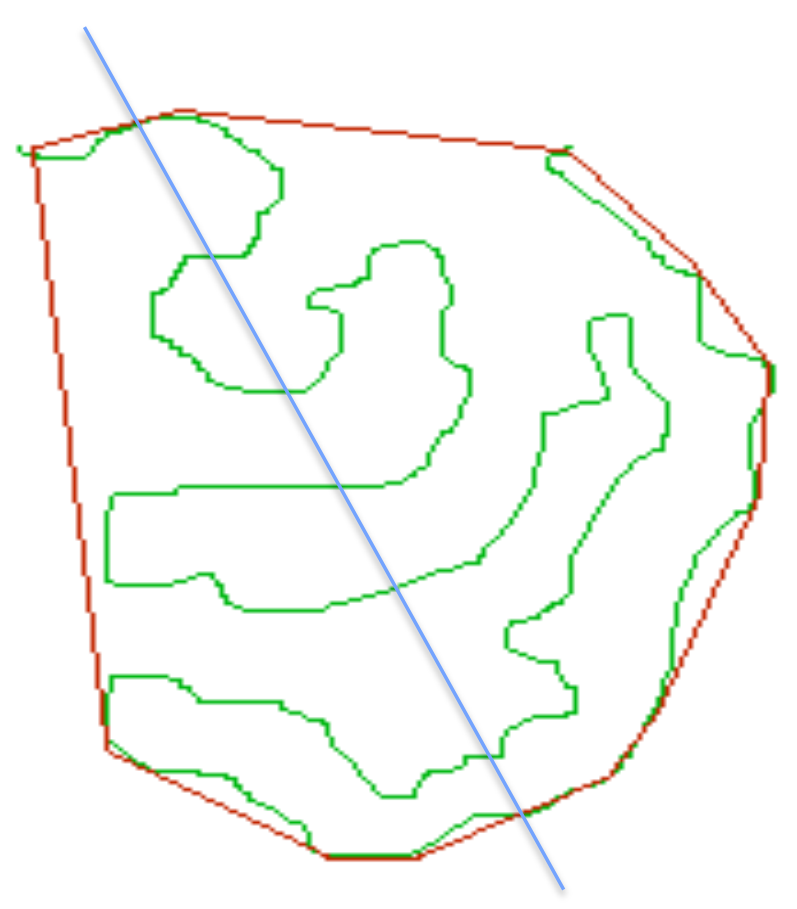


Fusing wiggly texture polygons to obtain object polygons
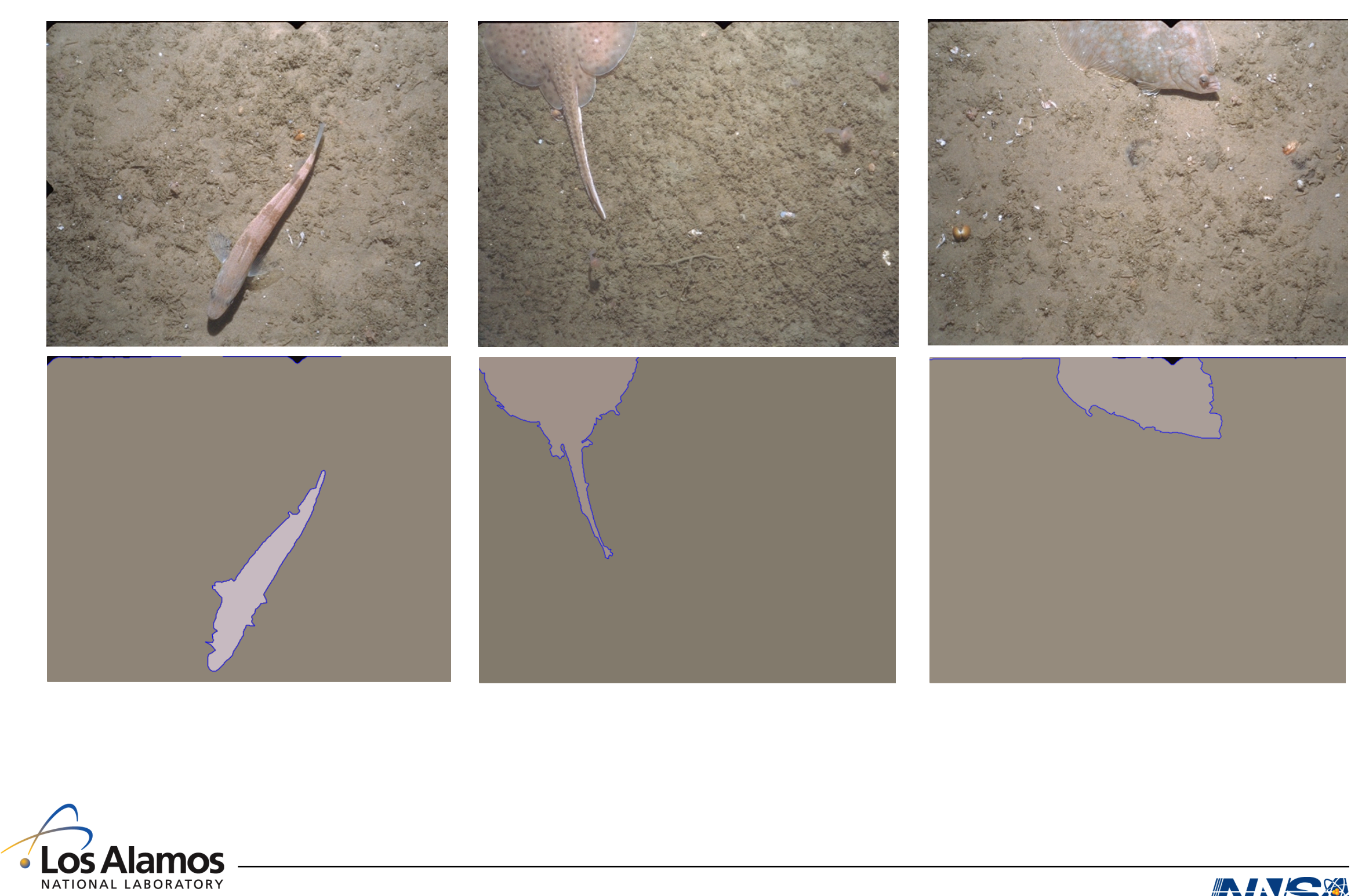


\section{Object Recognition: the shape of things...}

The 2-D relationships among a 1-D contour's points determine shape

Quest for a regional binding of contour elements

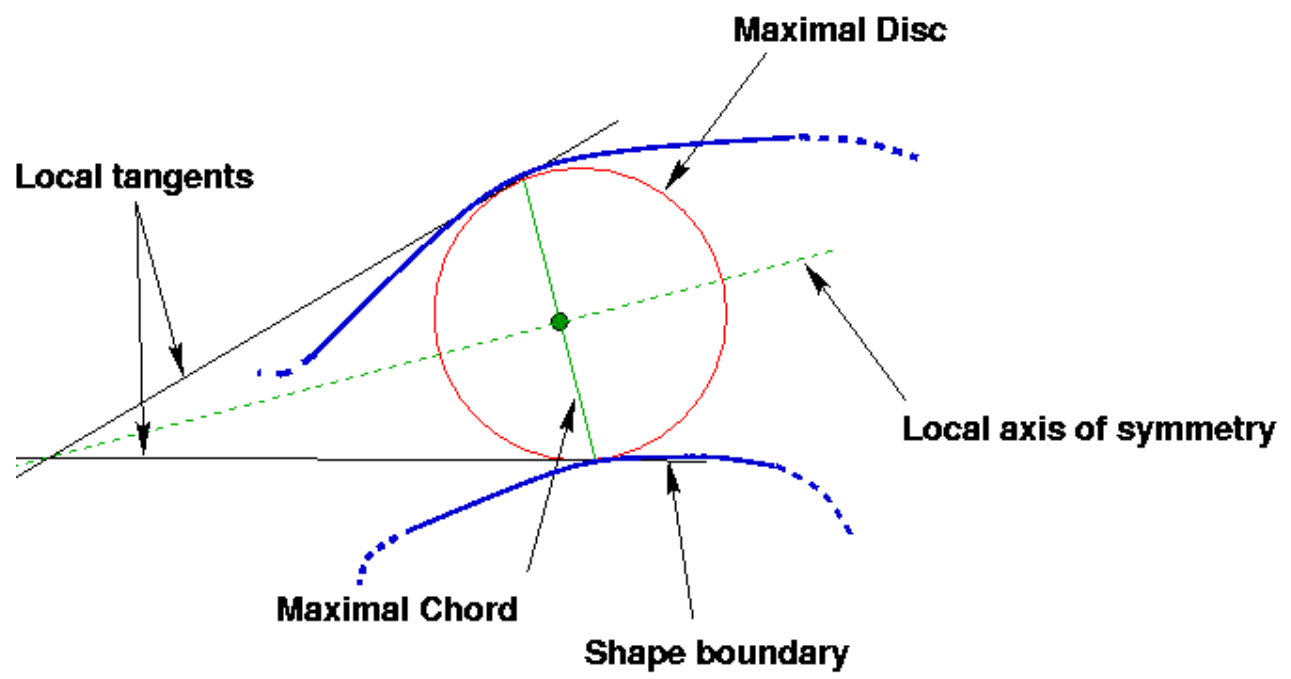

The medial axis transform (MAT) of a shape, which is the locus of centers of all maximal discs of the shape, is known to be highly unstable to small perturbations in the shape boundary 


\section{Generalizing to discretized shapes}

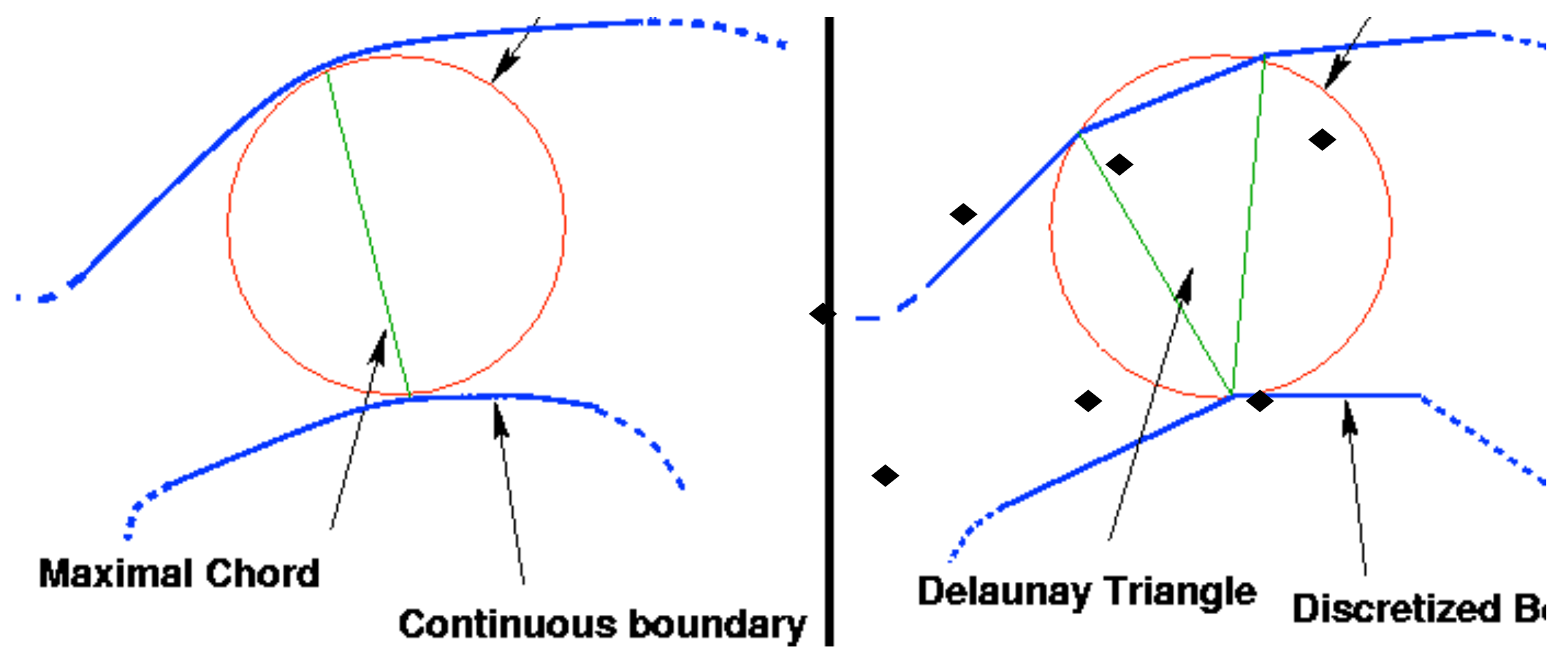

Internal edges of the Delaunay triangulation of a shape's interior approximate maximal chords for discretely sampled shapes 


\section{Constrained Delaunay Triangulation (CDT) of a Shape and Triangle Classification}

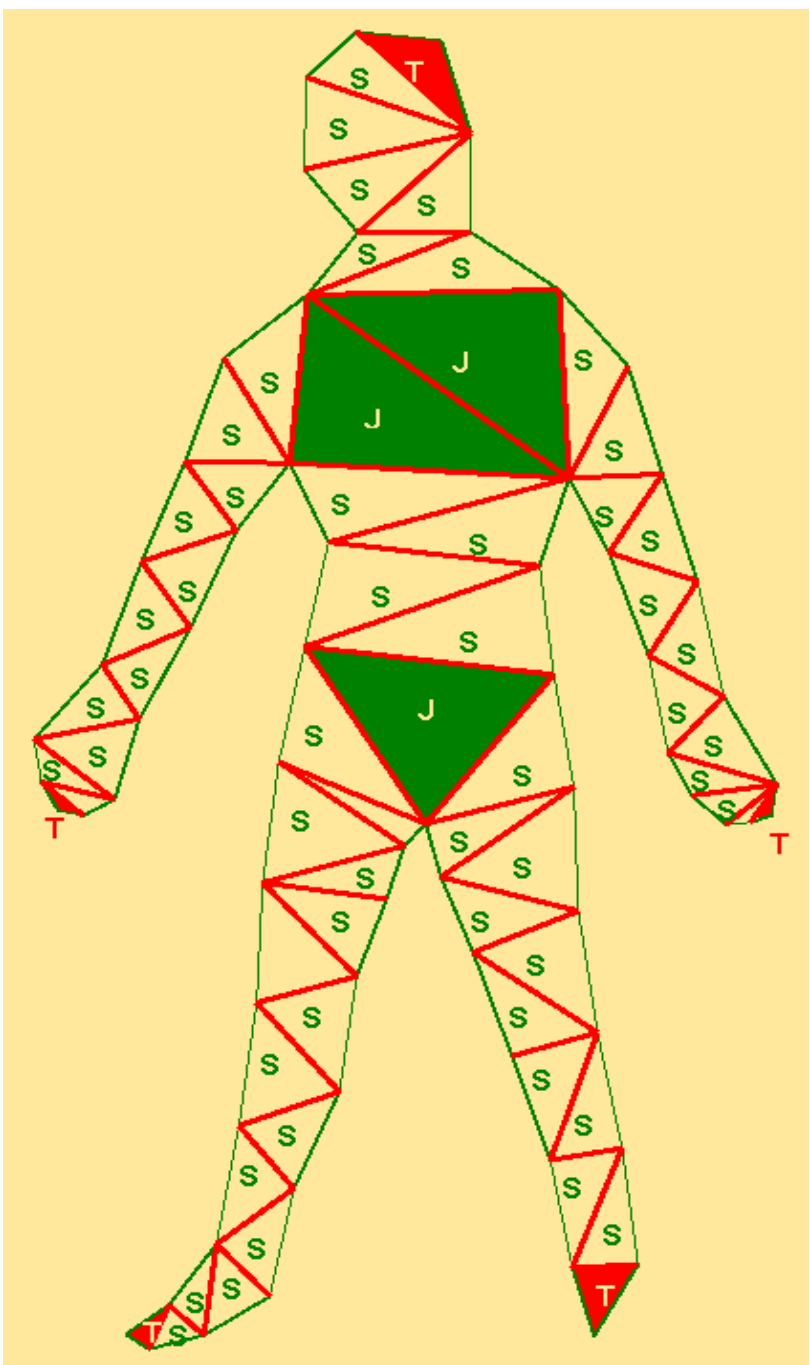

There are three kinds of triangles in a Shape's CDT representing different morphological events

Junction Triangles

no external edge; signify shape bifurcation

Sleeve Triangles

one external edge; signify shape prolongation

Terminal Triangles

two external edges; signify shape termination

This classification enables the segmentation of shapes into semantically salient parts. 


\section{The Chordal axis transform (CAT) of a shape with discretized boundary (Prasad et al SPIE 2000)}

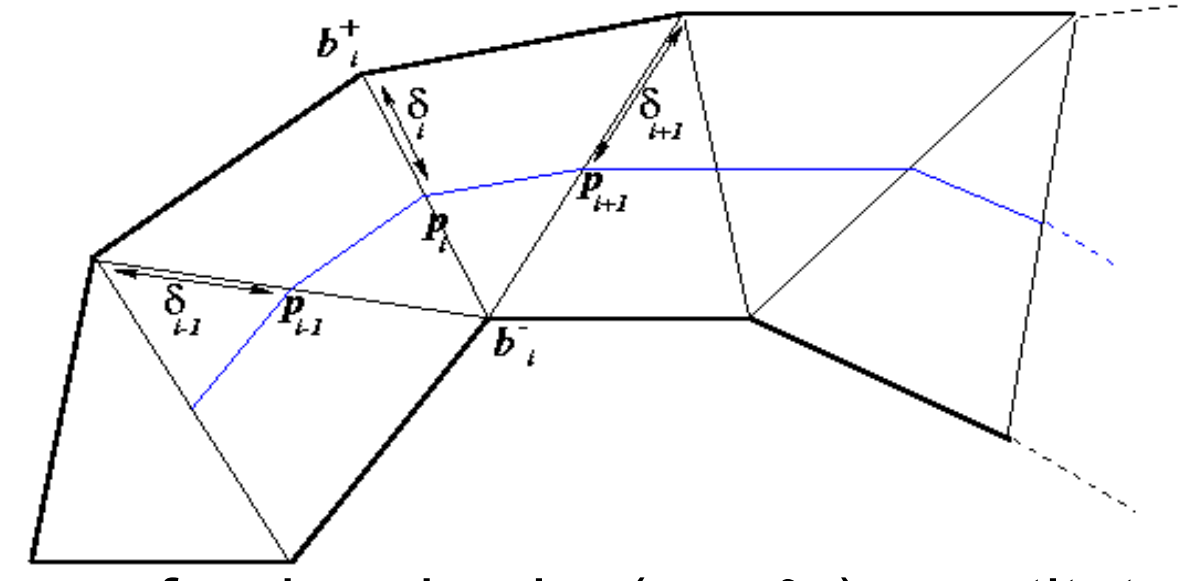

Sequences of ordered pairs $\left(p_{i}, \delta_{i}\right)$ constitute the CAT Prasad, Morphological Analysis of Shapes, LANL CNLS Newsletter, June 1997

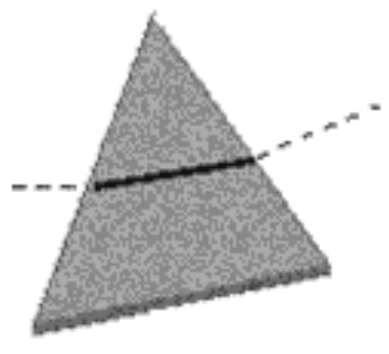

Sleeve

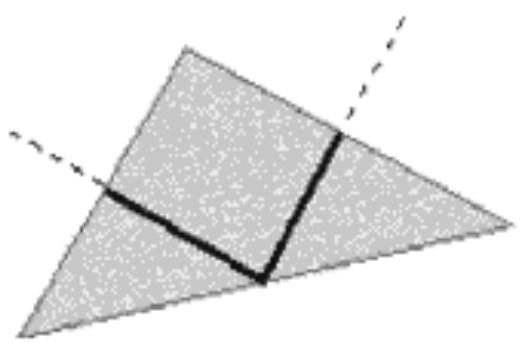

Obtuse Junction

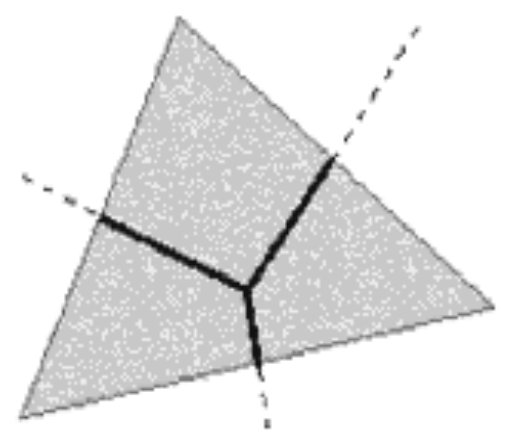

Acute Junction

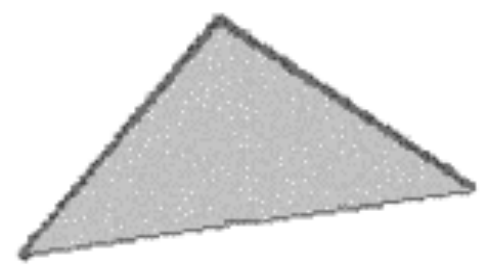

Terminal

Local CAT skeleton construction 


\section{Shape segmentation and stability of the CAT}

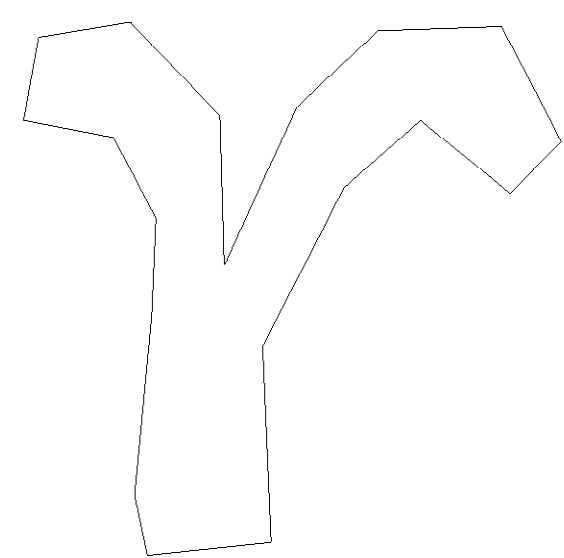

1. Polygonized Shape

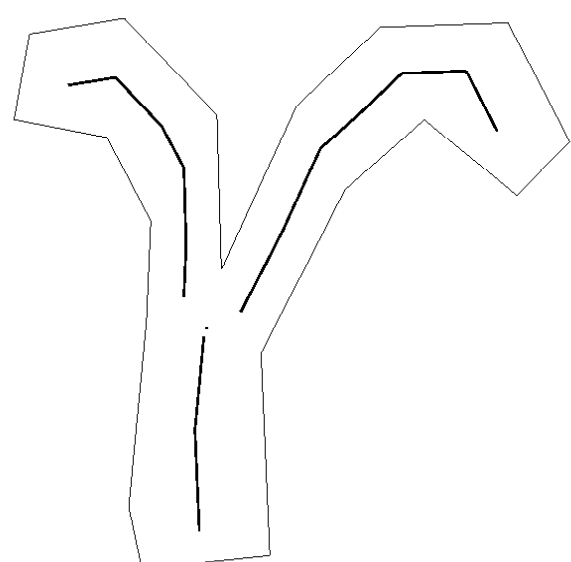

4. CAT Protoskeleton

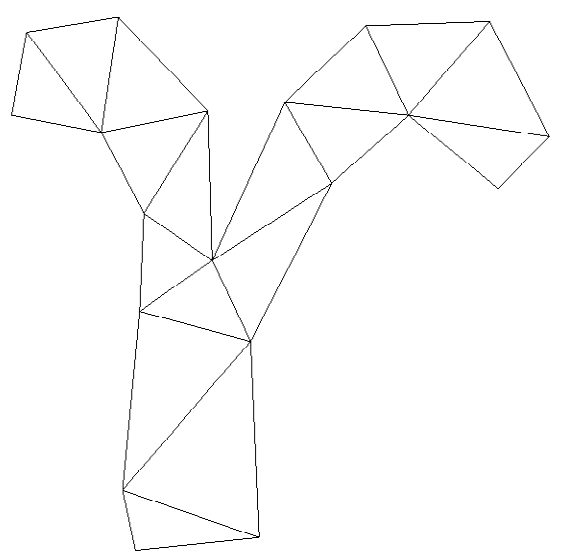

2. Delaunay Triangulation

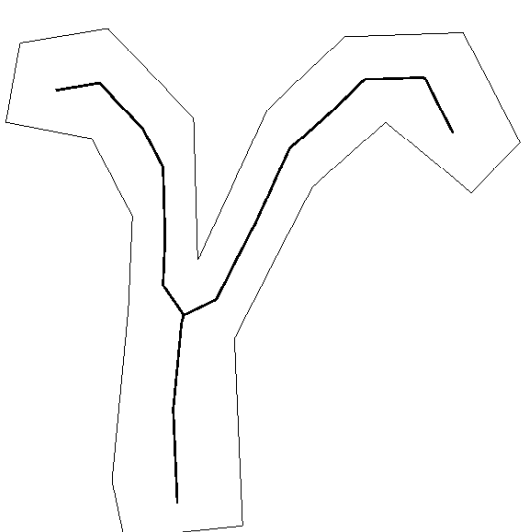

5. CAT Skeleton

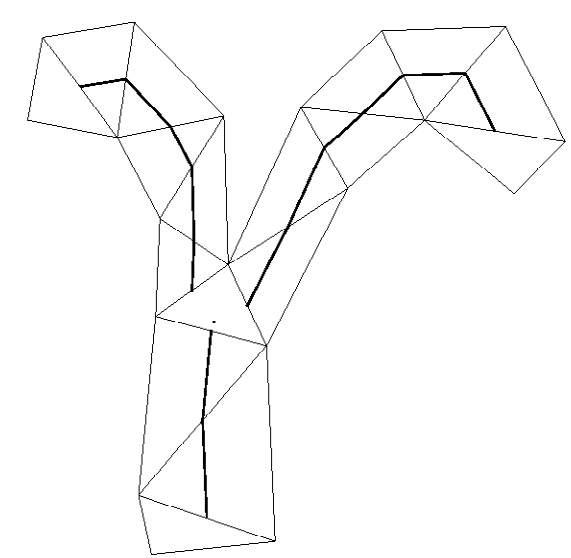

3. Segmentation by CAT

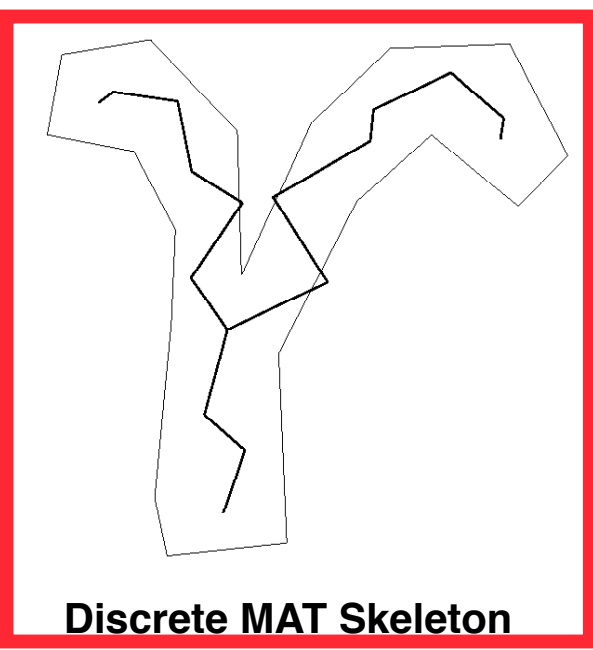




\section{Scale selectivity via a Morphological Pruning Criterion}
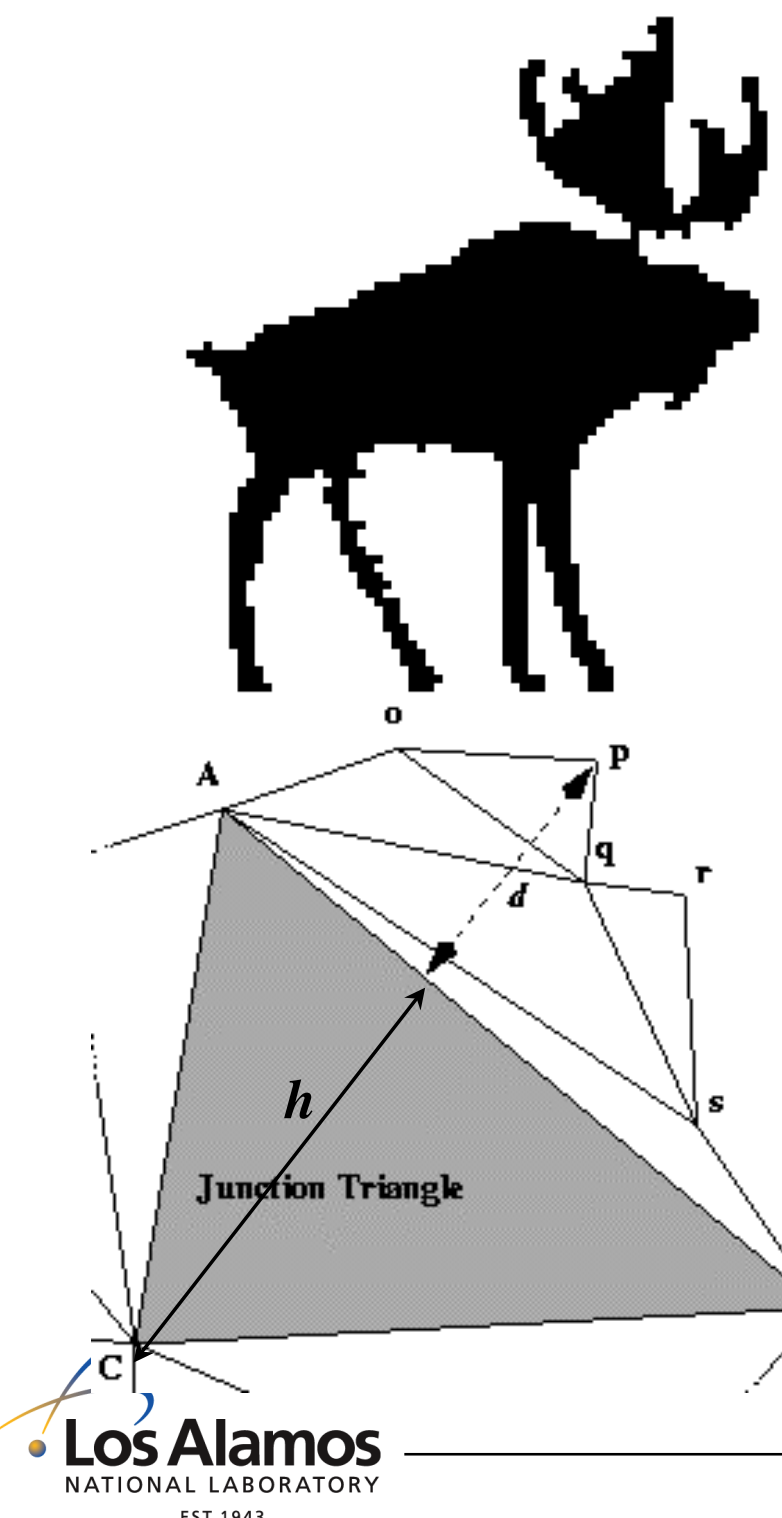
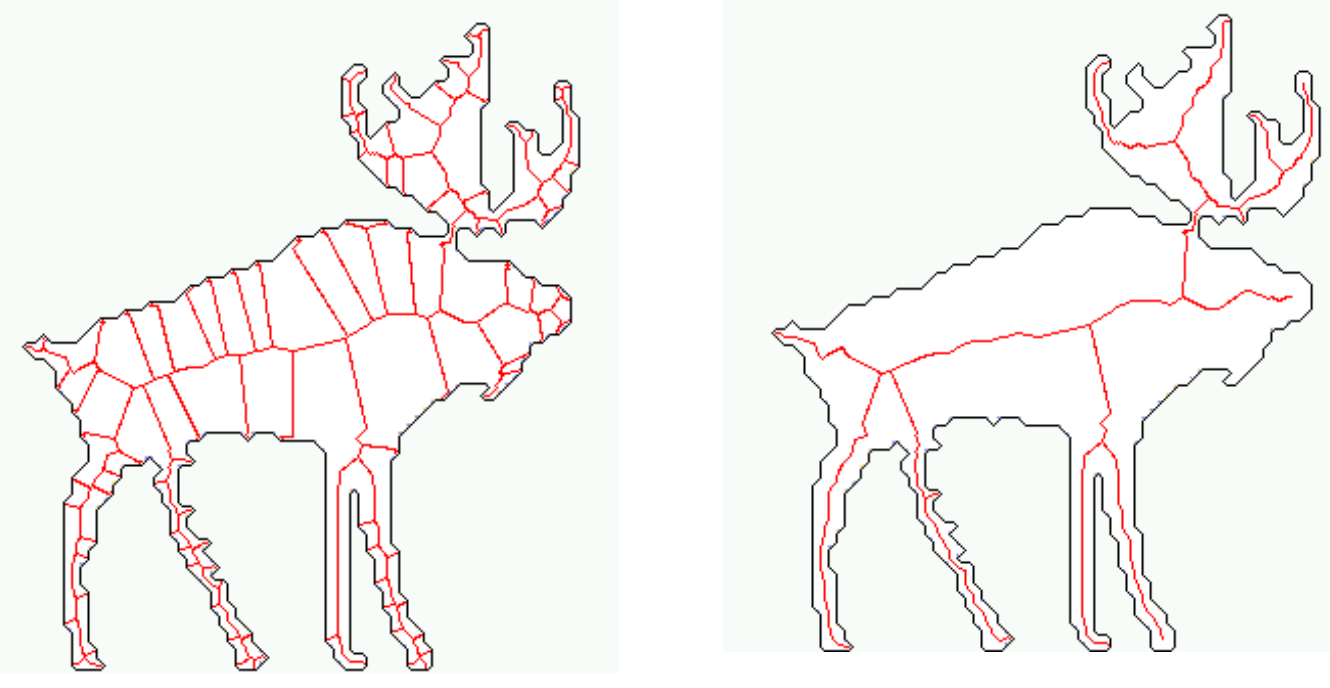

The ratio of morphological significance $\boldsymbol{d} / \boldsymbol{h}$ determines whether the shape segment AopqrsBA is to be excised or not 


\section{Delaunay Triangulation as an adaptive shape grid}

The Delaunay triangulation of a shape's interior is a natural morphological grid that helps analyze the shape's structure

A shape's complex global structure can be simply described in terms of local structural events such as bifurcations, terminations and prolongations

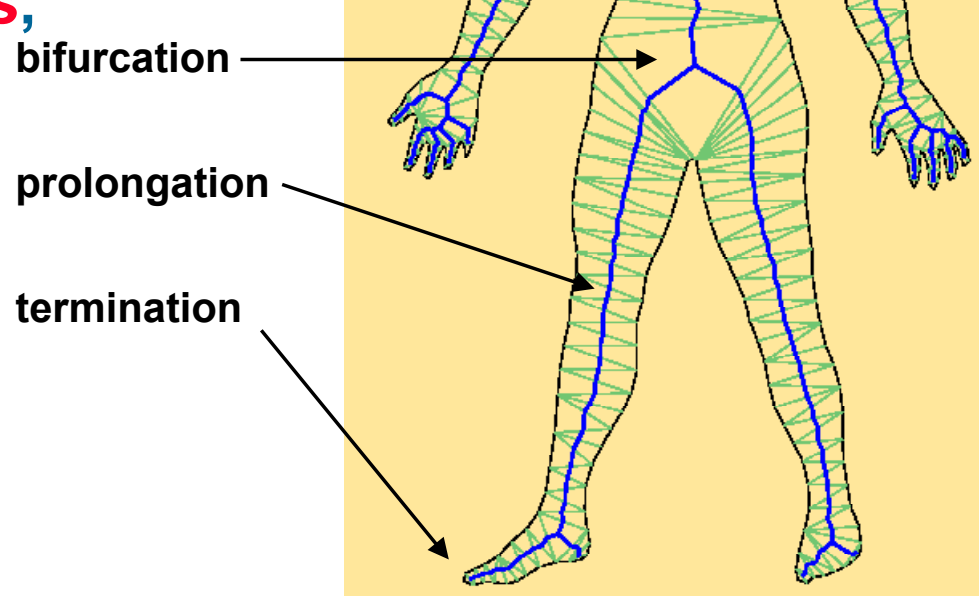




\section{Feature Identification via Delaunay Triangulation}

Identify two kinds of triangular chain complexes:

Limb - A chain complex of the form TS...SJ

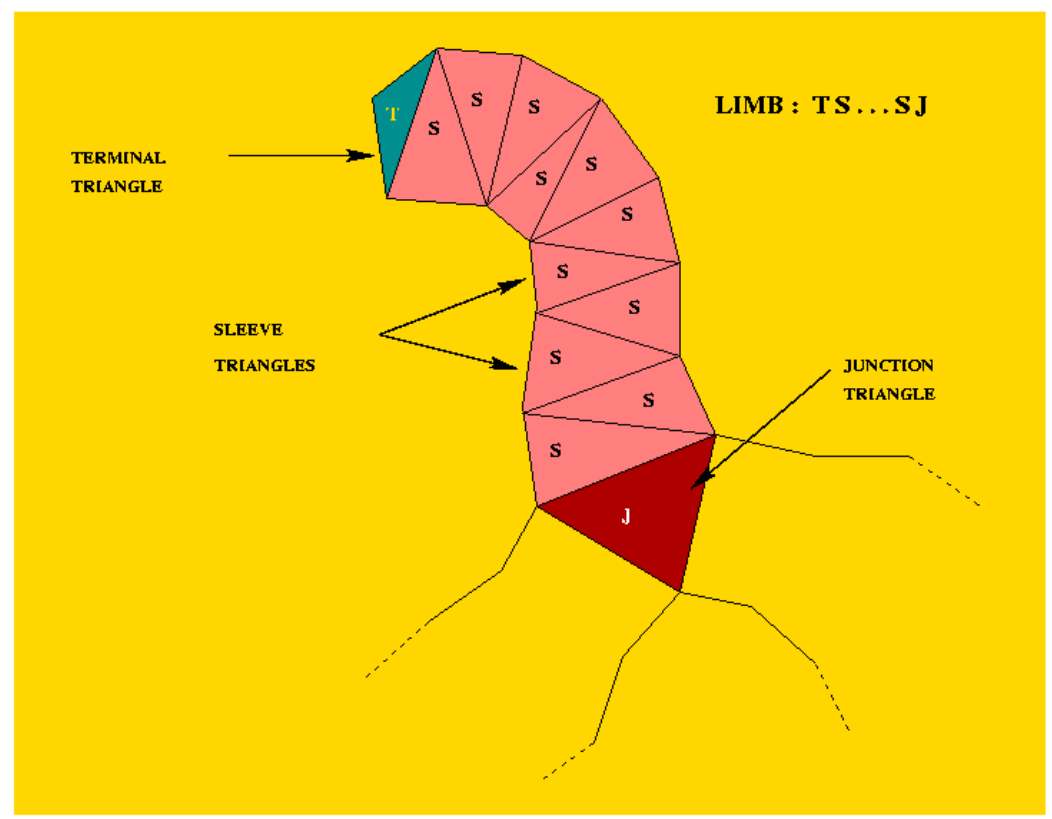

Torso - A chain complex of the form JS...SJ

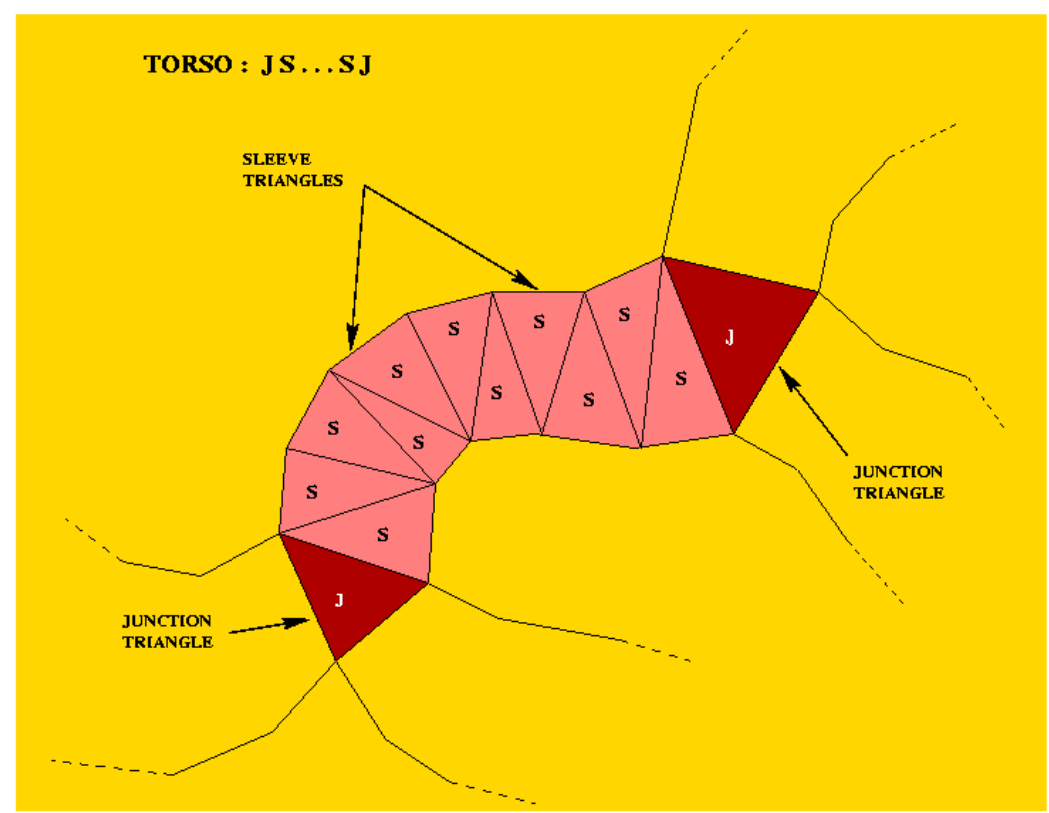




\section{Chord Strength and salient region-contour bindings}

For each internal triangle edge (chord) $e$ we define the strength of $e$ as the valuation

$$
f(e)=1-\left(\theta_{e}+\varphi_{e}\right) / \pi,
$$

where $\theta_{e}$ and $\varphi_{e}$ are the angles opposite the edge $\mathrm{e}$ in its two flanking triangles

1-f is a measure of the cocircularity of the triangles flanking $e$

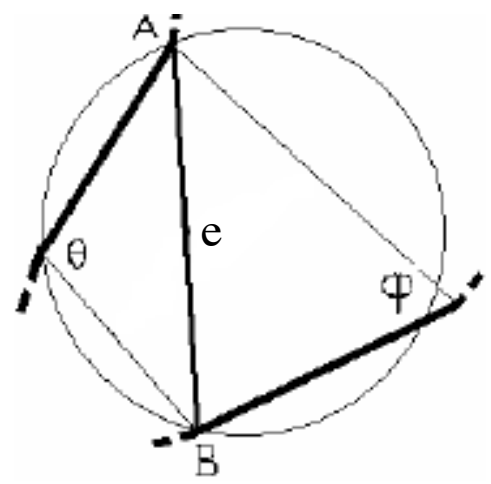

Flipping the common edge e of cocircular adjacent triangles does not violate the Delaunay criterion, but can produce different local skeletons

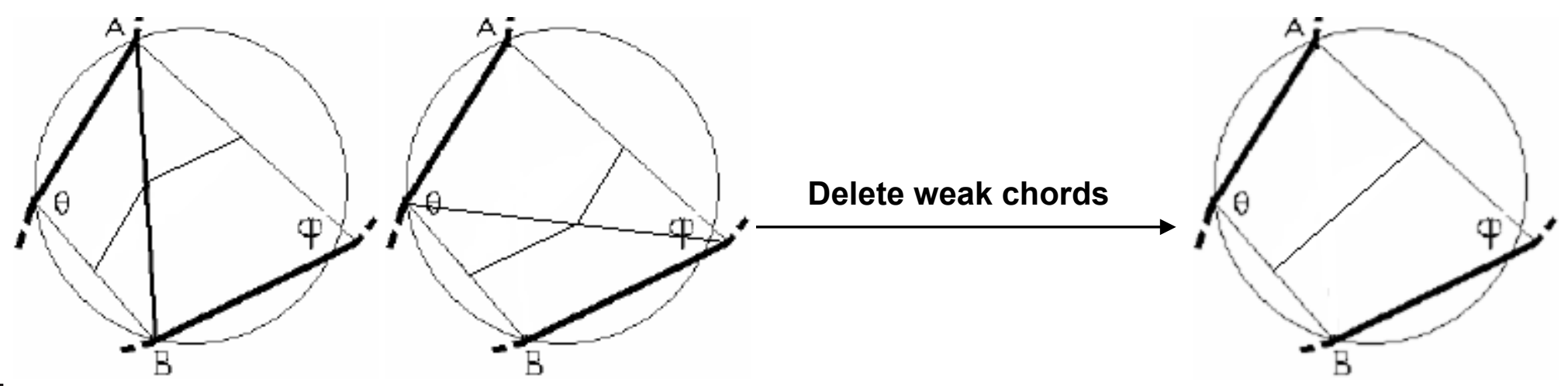




\section{Retention of strong chords}

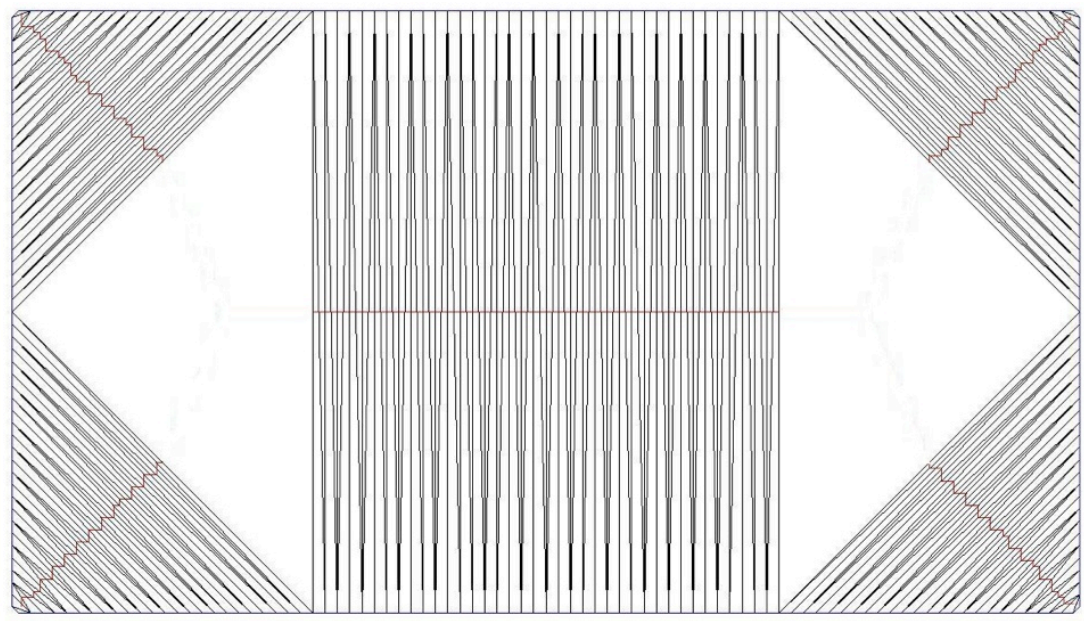

CAT protoskeleton induced by all chords

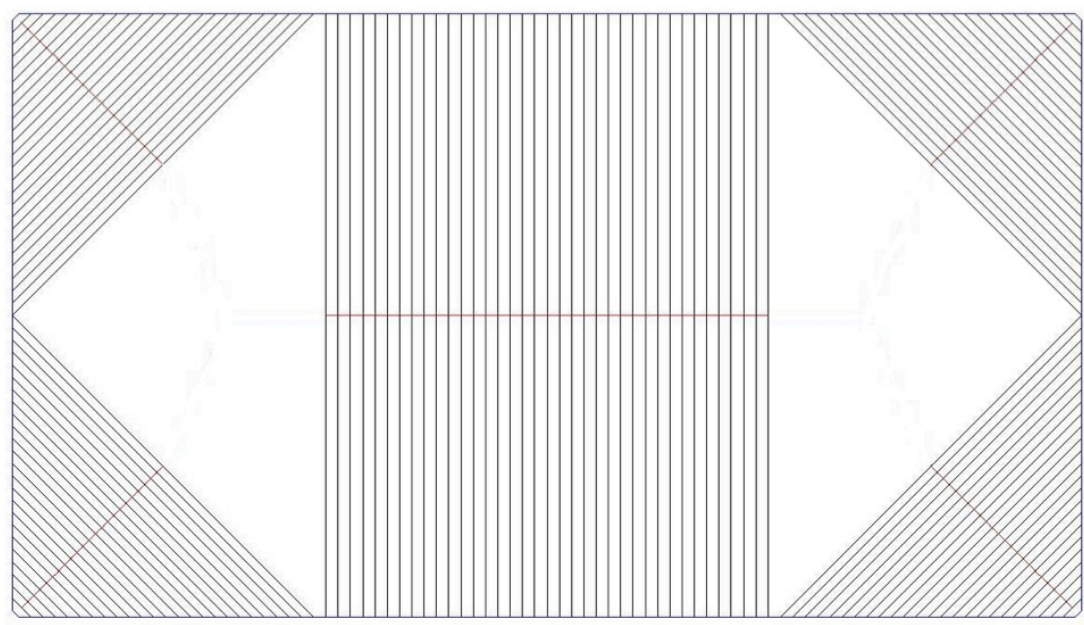

CAT protoskeleton induced by strong chords
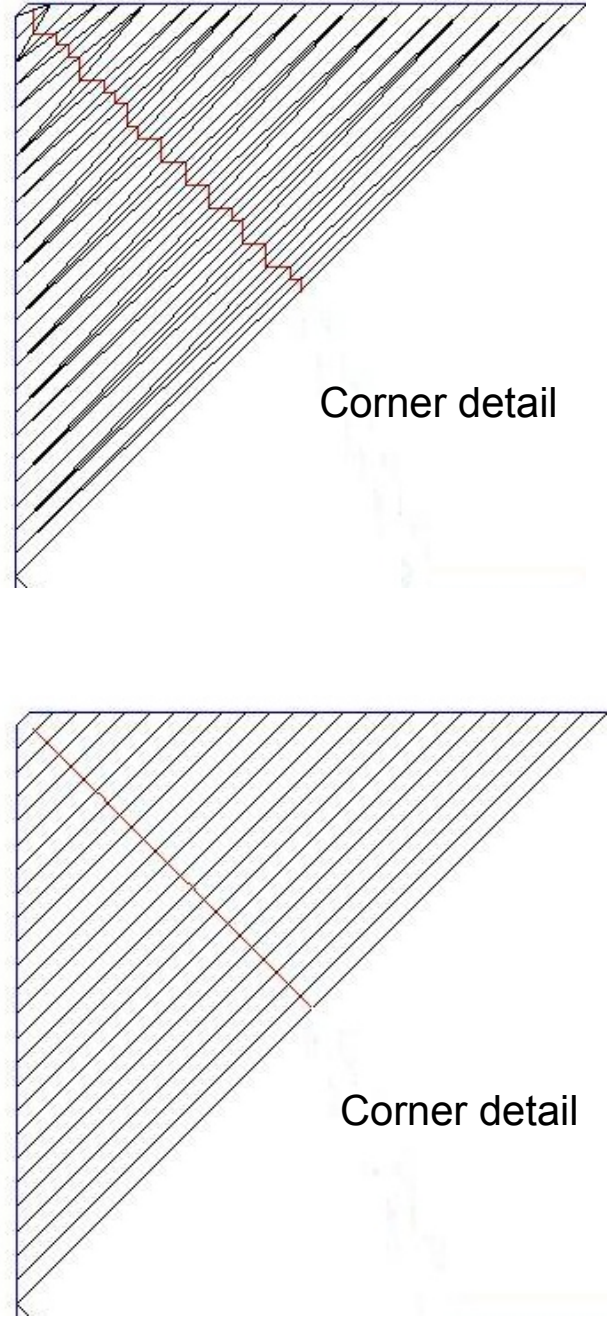


\section{The CAT and the psychophysics of human vision}

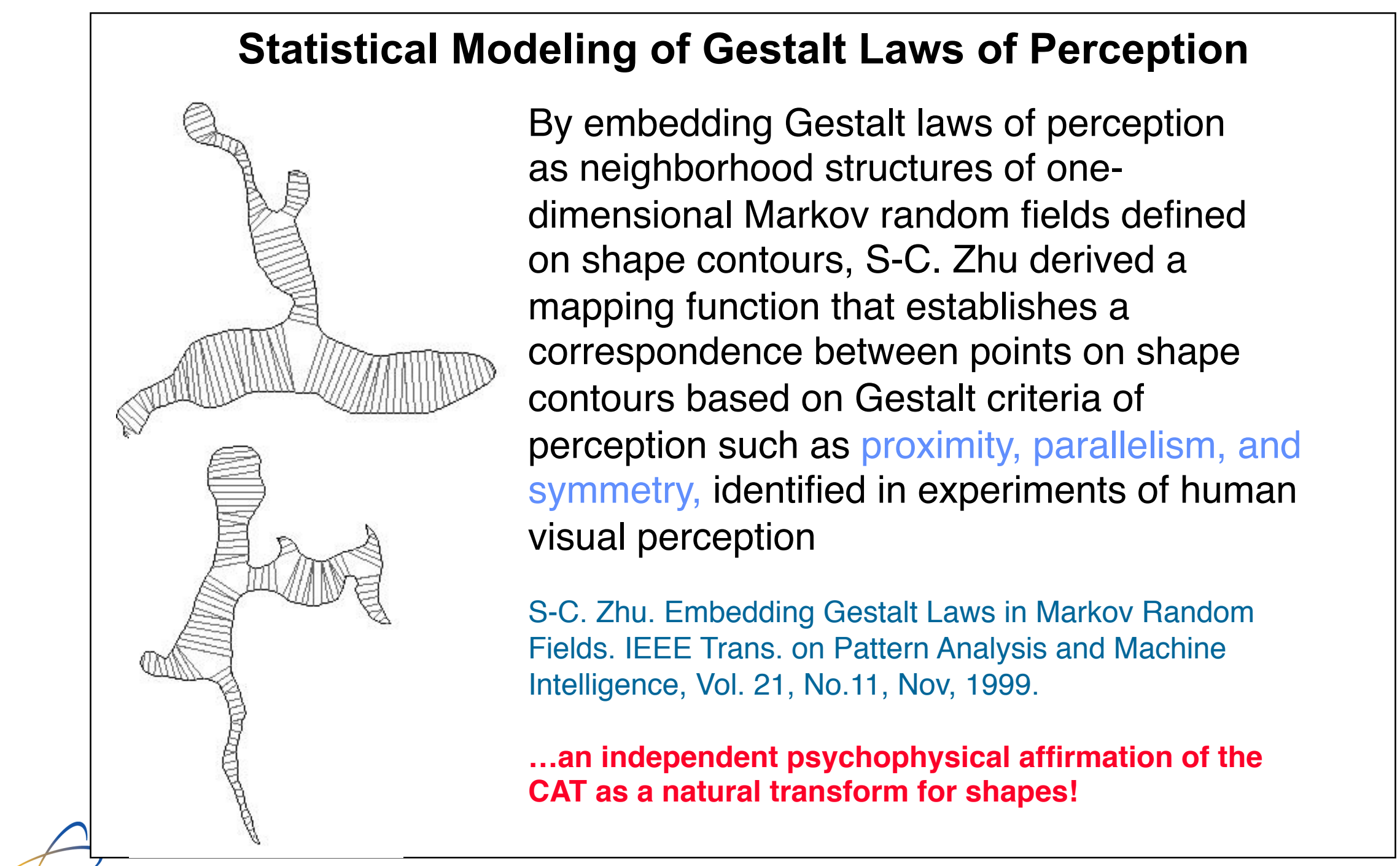




\section{CAT and higher degree branch points}

Elimination of weak chords creates polygons whose vertices are approximately co-circular points.

This creates branch points of degrees $>3$ three that are stable under small shape deformations.

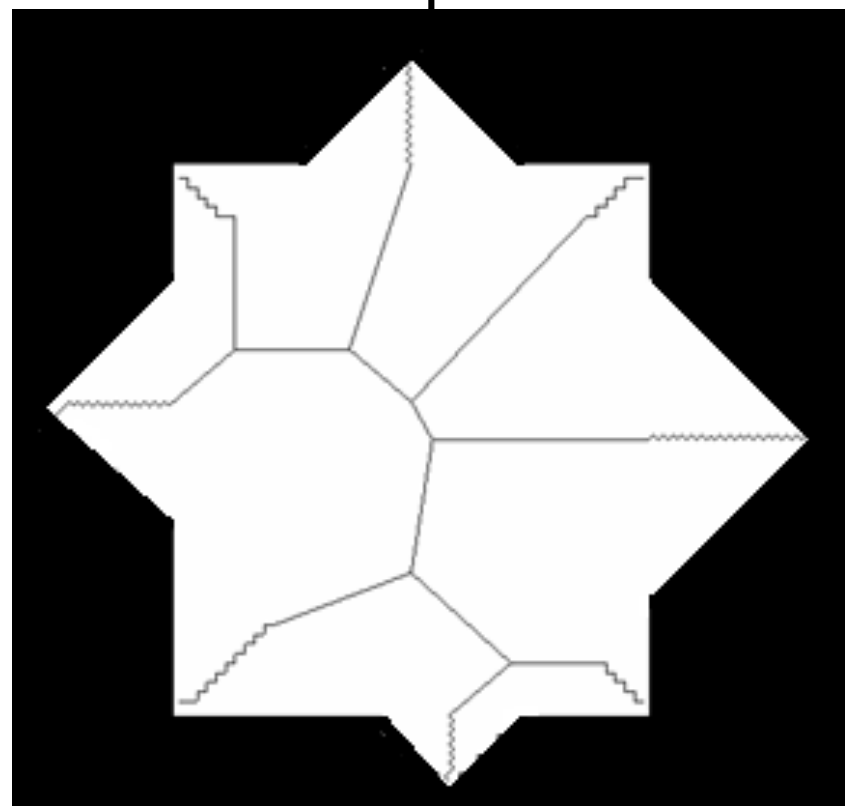

CAT

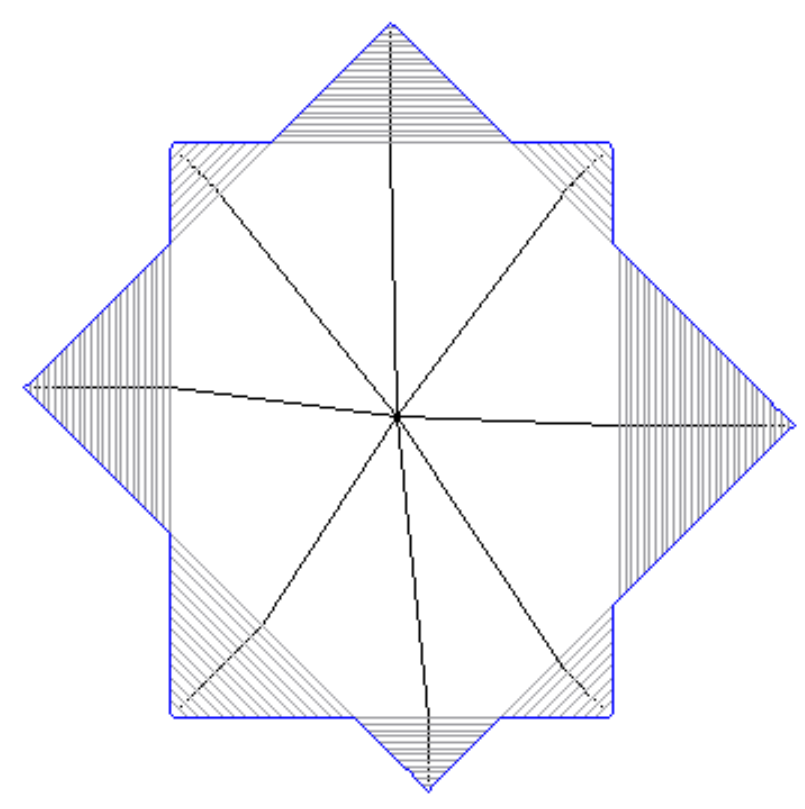

Rectified CAT

L. Prasad, Rectification of the Chordal Axis Transform and criteria for shape decomposition, Image and Vision Computing, vol. 25, Issue 10, 2007 


\section{Examples of CAT skeletons of some shapes}
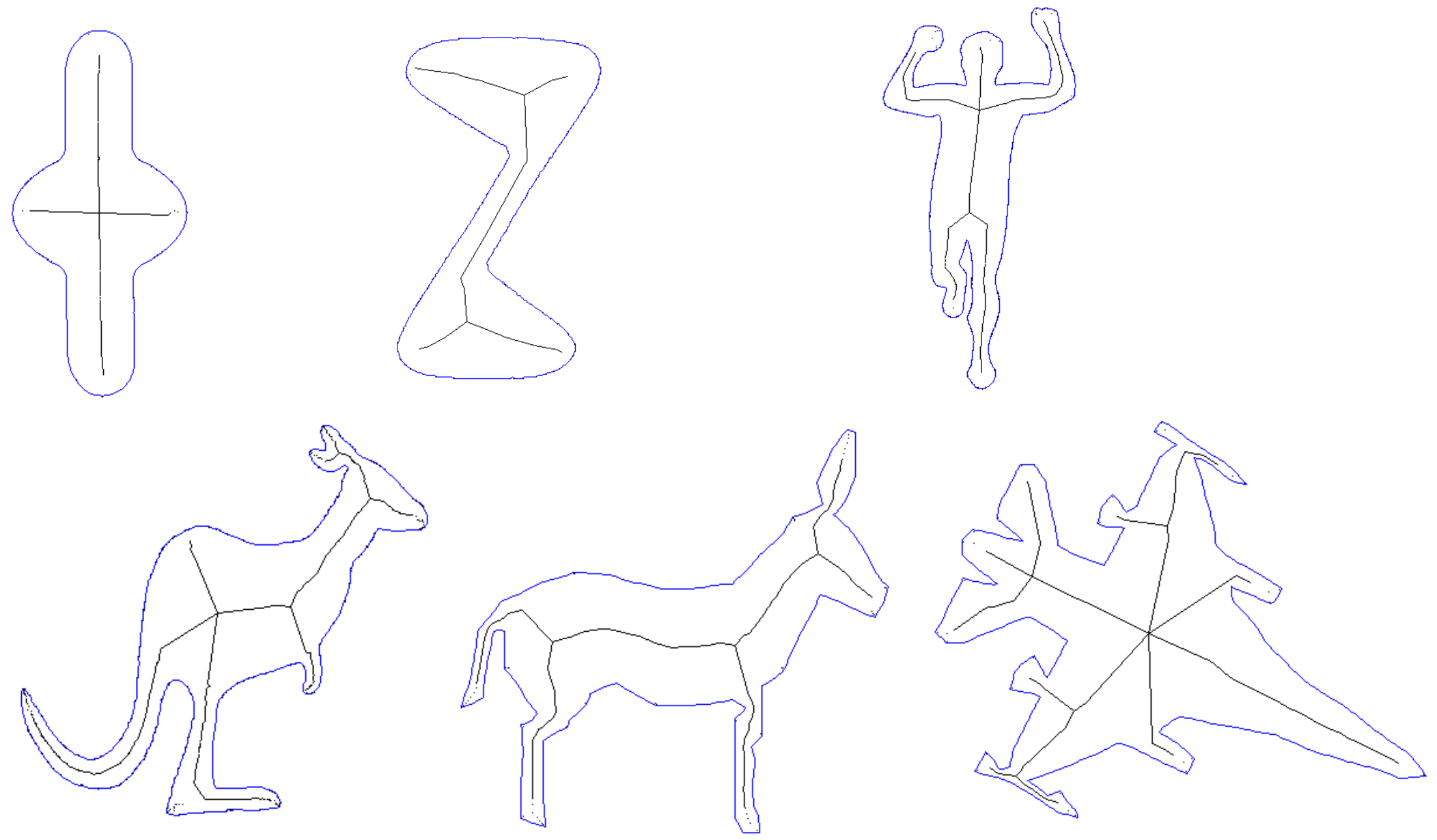


\section{Decomposition of shapes into visual parts:}

The axial shape derivative (Prasad, Image \& Vision Computing, 2007)

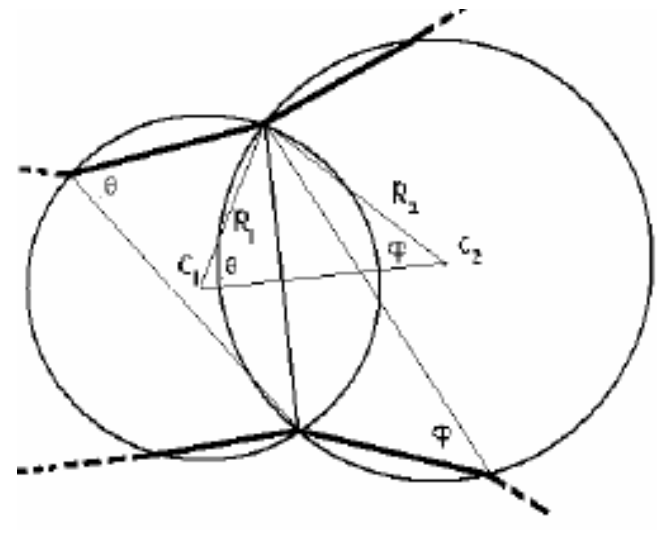

$$
\frac{\Delta R}{\Delta c}=\frac{R_{2}-R_{1}}{c_{2}-c_{1}}=\frac{\sin \left(\frac{\theta-\varphi}{2}\right)}{\sin \left(\frac{\theta+\varphi}{2}\right)}
$$
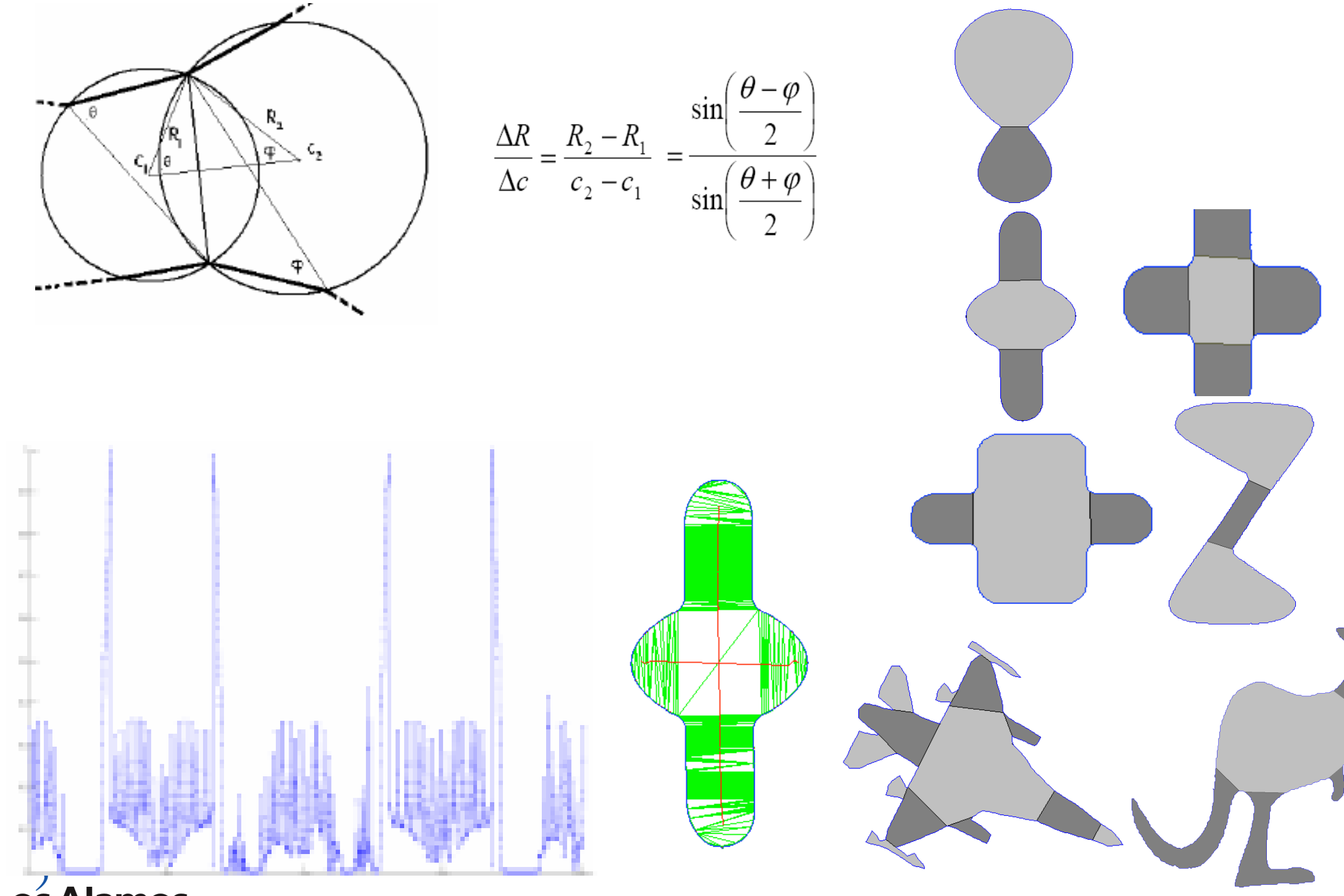


\section{Decomposition of shapes into visual parts:}

The axial shape derivative (Prasad, Image \& Vision Computing, 2007)

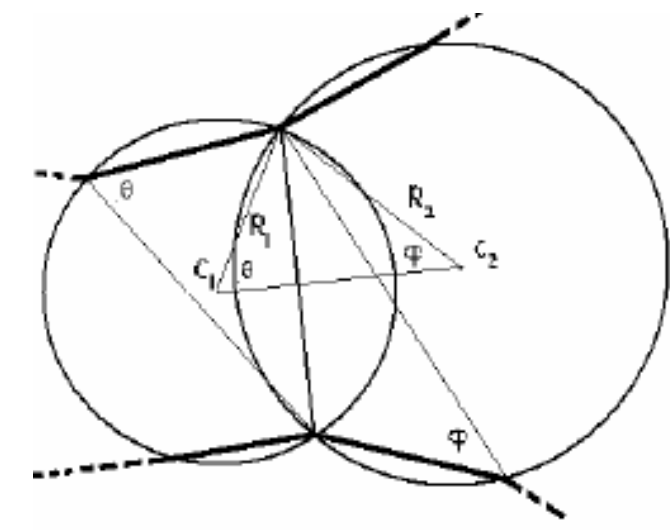

$\frac{\Delta R}{\Delta c}=\frac{R_{2}-R_{1}}{c_{2}-c_{1}}=\frac{R_{2}-R_{1}}{R_{2} \cos \varphi+R_{1} \cos \theta}=\frac{\sin \theta-\sin \varphi}{\cos \varphi \sin \theta+\cos \theta \sin \varphi}=\frac{\sin \left(\frac{\theta-\varphi}{2}\right)}{\sin \left(\frac{\theta+\varphi}{2}\right)}$
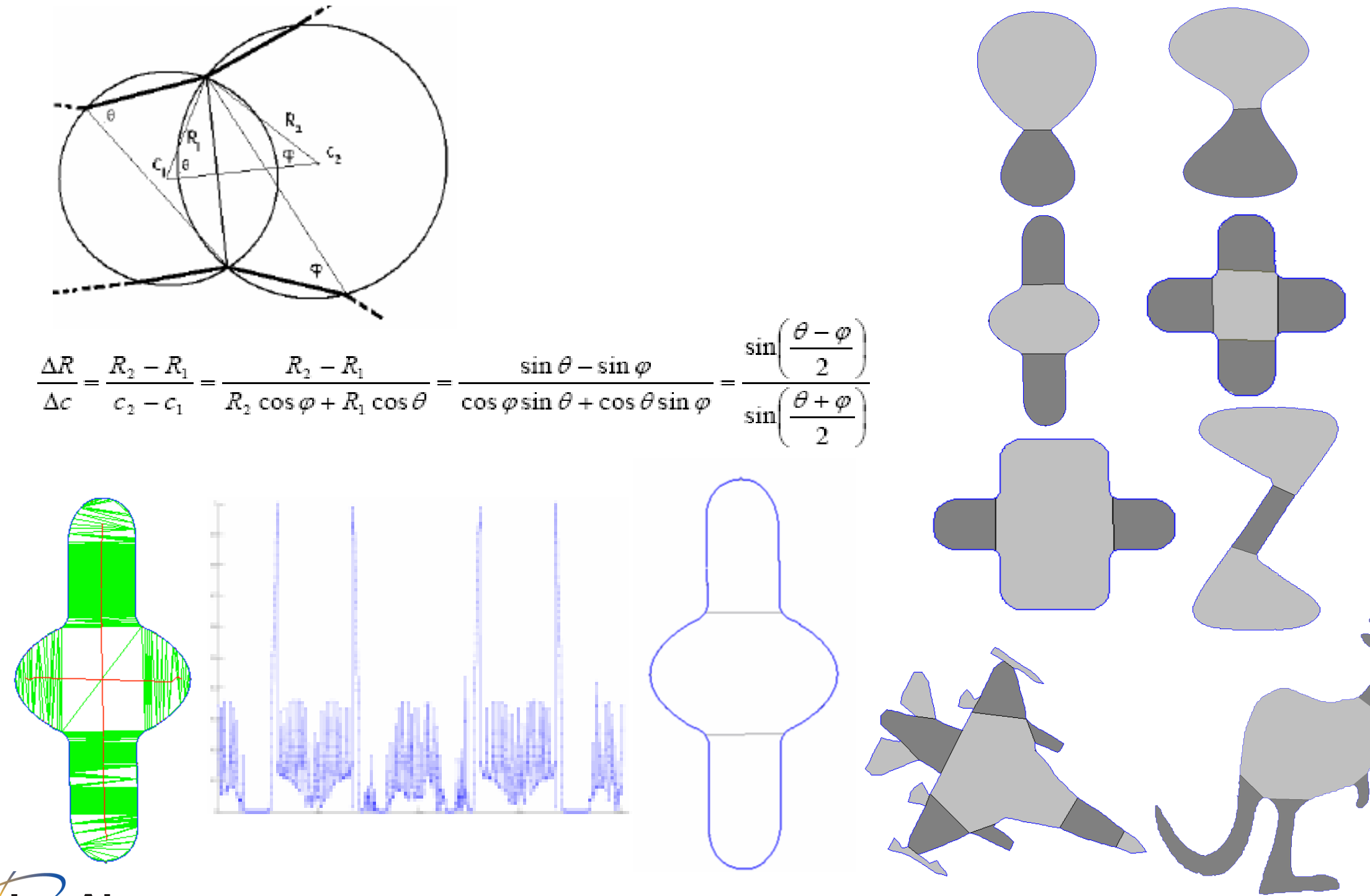
A Generative Context-Free Grammar for Shapes (Prasad et al SPIE 2000)

$\boldsymbol{G}=\left(\{S, L, H\},\left\{I_{0}, h_{0}, I, h,(),\right\}, S, \boldsymbol{R}\right)$ The rule set $\boldsymbol{R}$ :

$S \longrightarrow I_{0} \quad$ String generator

$S \longrightarrow h_{0} \quad$ Loop generator

$L \longrightarrow I \quad$ Limb terminator

$H \longrightarrow h$ Handle terminator

$S \longrightarrow \begin{array}{ll}L L L \\ S H \quad \text { Shape initiators }\end{array}$

$S \longrightarrow H H$

$\stackrel{L \longrightarrow 7}{L} \begin{gathered}L L \\ (L L)\end{gathered}$

$L \longrightarrow-(H)$

Limb, torso \& handle generators

$\mathrm{H} \longrightarrow \mathrm{HH}$

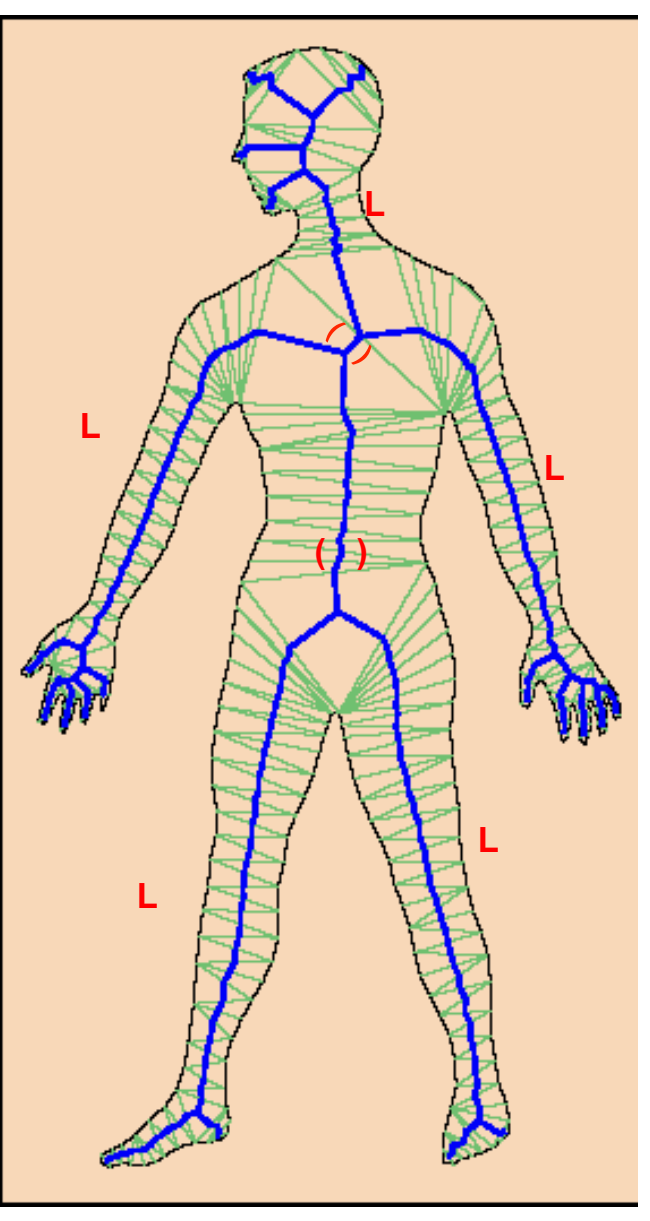

$\mathrm{L}(\mathrm{L}(\mathrm{LL})) \mathrm{L}$

$\mathrm{H} \longrightarrow \mathrm{HLH}$

Grammar rules, applied in reverse, enable multiresolutional shape description: 


\section{Multiresolutional Syntactic Representation of a Shape}

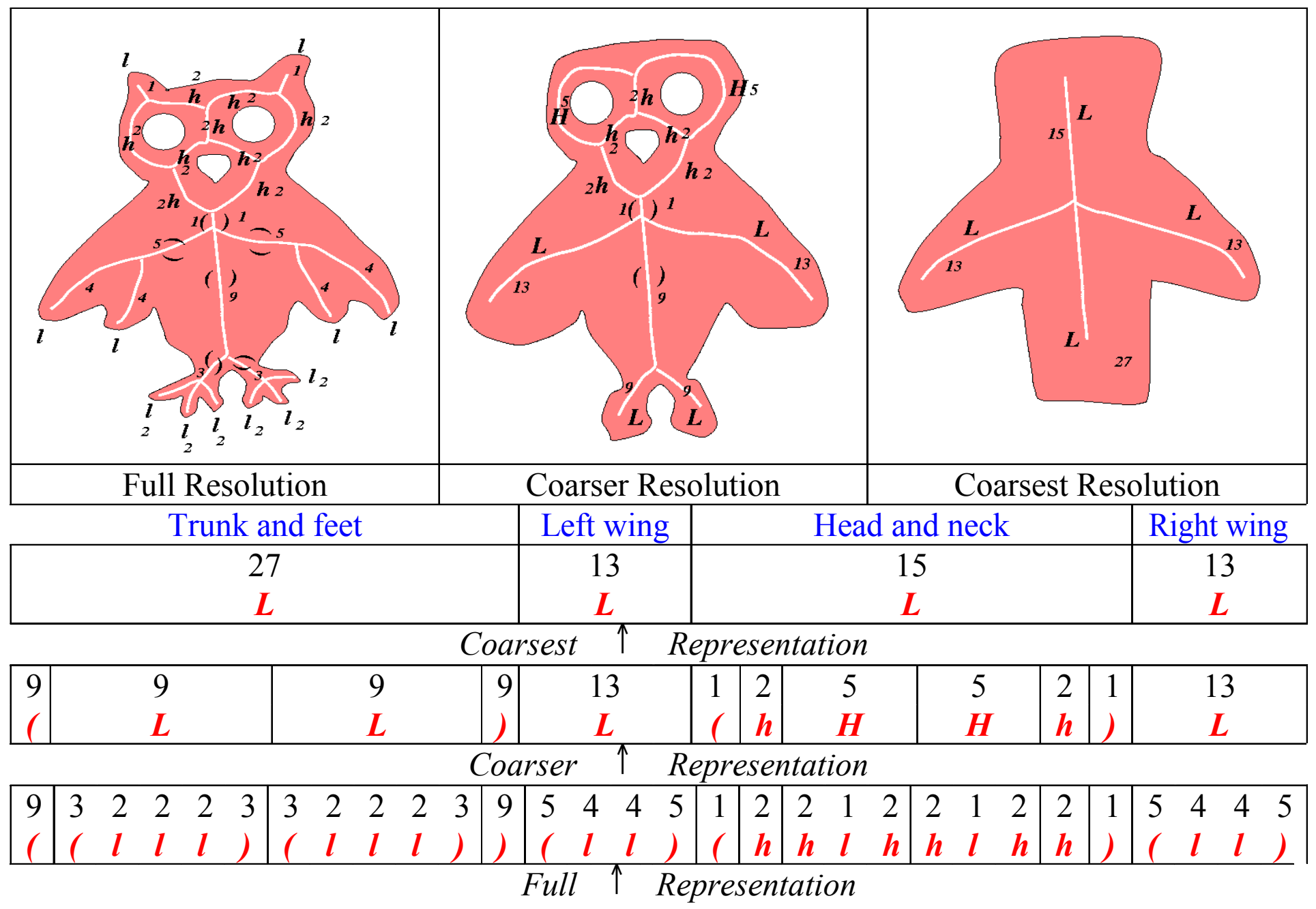




\section{Shape Recognition}

- This linguistic representation establishes a flexible computational language of shapes that allows for both "ballpark" as well as precise description.

- The hierarchical representation of shapes via linguistic string templates enables the design of complex databases for shape recognition.

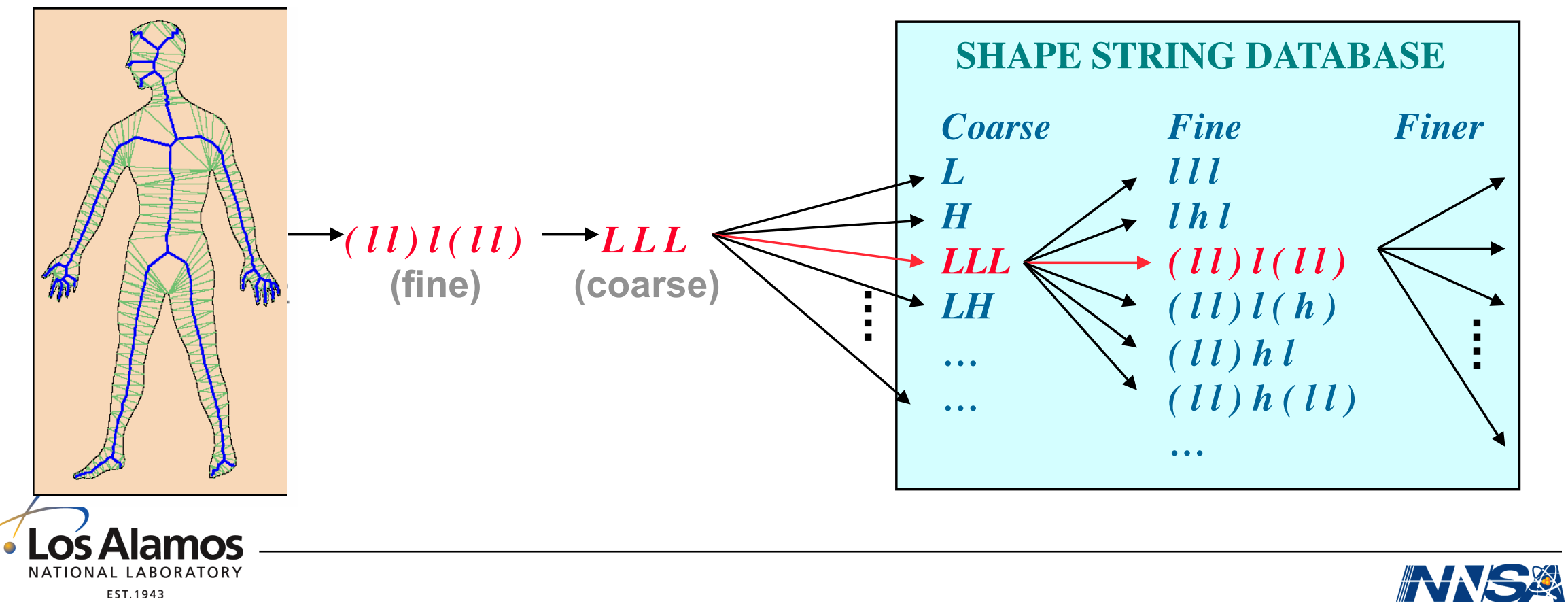


Linguistic shape characterization and detecting symmetry via straight skeleton segments: Manmade vs. Natural Objects
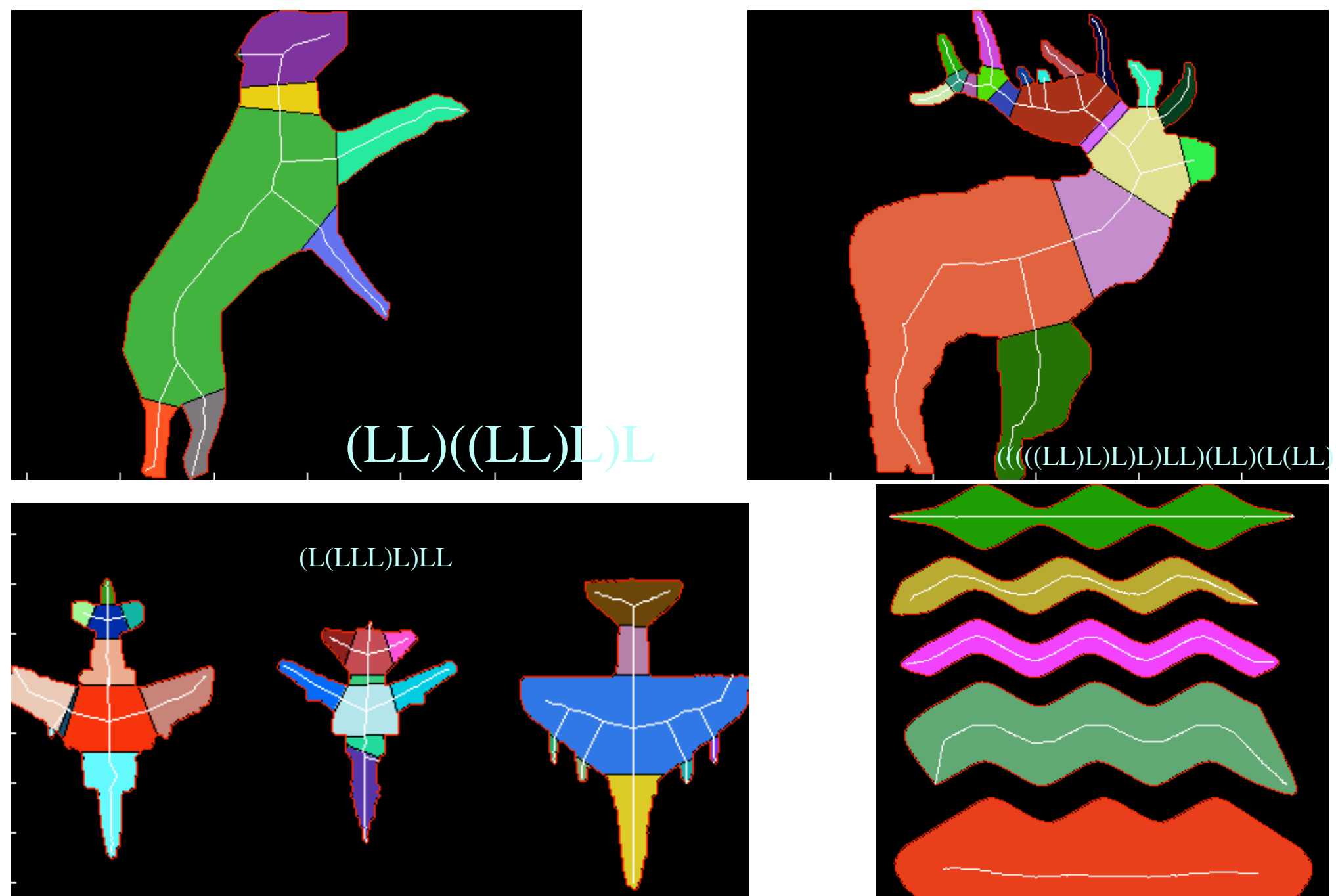

(LL(LL))LLL

$((\mathrm{LL}) \mathrm{L}) \mathrm{L}(\mathrm{L}(\mathrm{LL}))(\mathrm{LL})$

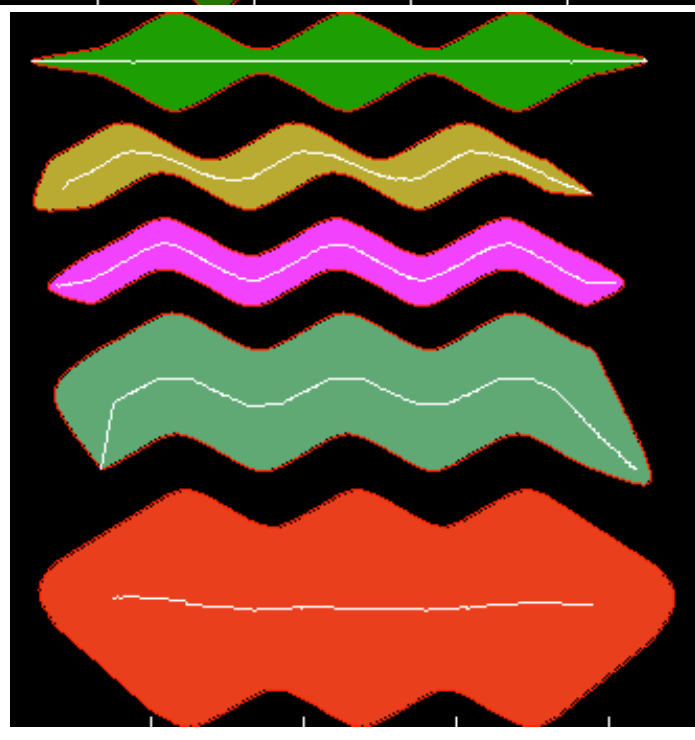




\section{Eliminating Rotational Ambiguity in the Syntactic Representation of a Shape}

Syntactic representation of a shape: $\quad \Sigma=\mathrm{C}_{1}\left(\mathrm{C}_{2}\right) \mathrm{C}_{3}\left(\mathrm{C}_{4}\right)$ here the $\mathrm{C}_{i}$ represent substrings

Some equivalent representations:

$$
\begin{aligned}
& \Sigma=\mathrm{C}_{2}\left(\mathrm{C}_{3}\left(\mathrm{C}_{4}\right) \mathrm{C}_{1}\right) \\
& \Sigma=\left(\mathrm{C}_{1}\left(\mathrm{C}_{2}\right) \mathrm{C}_{3}\right) \mathrm{C}_{4}
\end{aligned}
$$

Rotational ambiguity is eliminated by "balancing" the string with respect to its attributes.

This is done by ensuring that no substring within parentheses has its cumulative attribute more than half the cumulative attribute of the whole string 


\section{From Pixels to Syntactic Characterization}

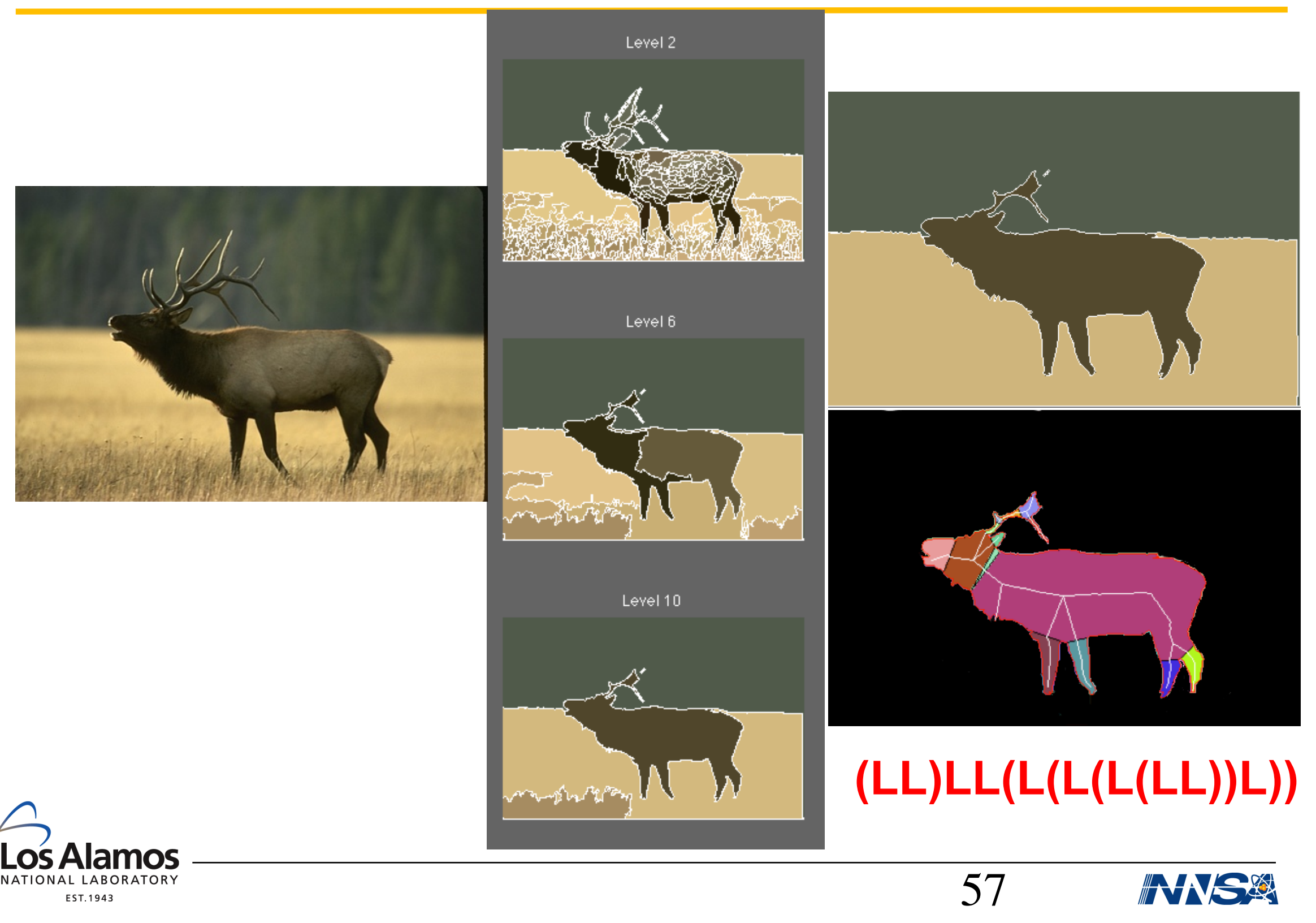




\section{Summary}

We have attempted to develop a unified framework for

- Efficiently computing a sparse, perceptually salient, hierarchical representation of images

- Characterizing and recognizing complex shapes and identifying their visual parts

Ullman' s criteria-a recap:

- Must support abstraction

capable of going from a low-level to high-level of representation

- Must be open-ended

i.e., support multiple visual routines

- Must be able to handle complexity

should deal with real world imagery

- More work to be done:

$>$ improve the modeling of perceptual criteria

$>$ infer contextual information of polygons in terms of spatial arrangement, scale, and saliency hierarchy for scene understanding 


\section{Ingredients so far: Frogs and snails and...}

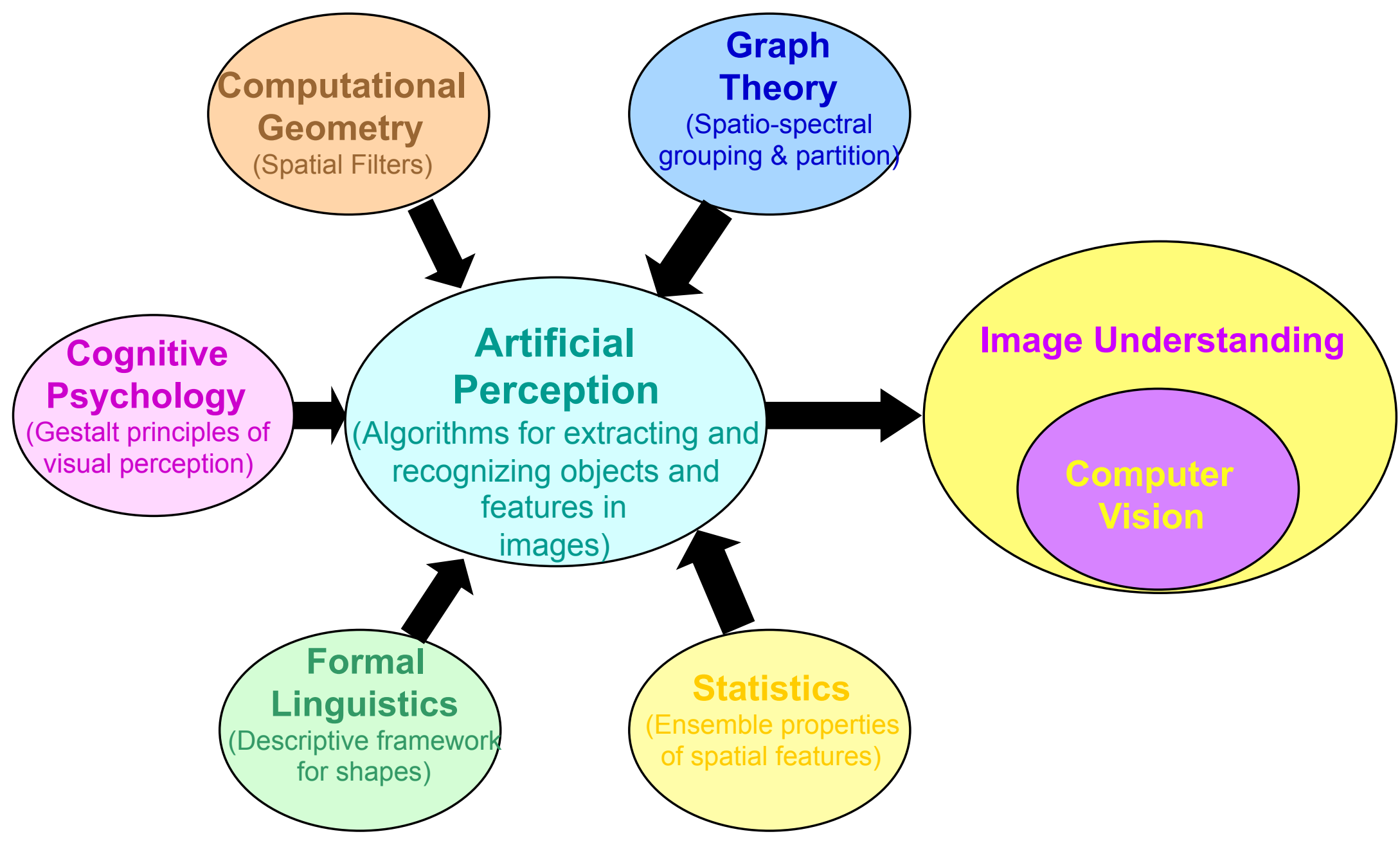

